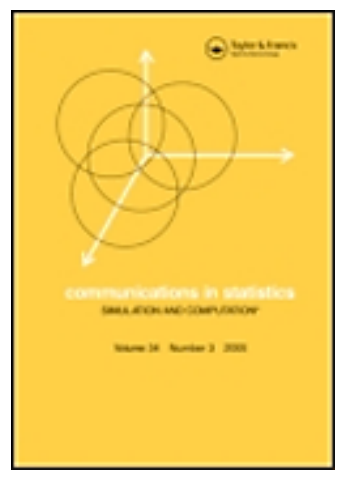

\title{
Reduced-Bias Location-Invariant Extreme Value Index Estimation: a Simulation Study
}

\begin{tabular}{|c|c|}
\hline Journal: & Communications in Statistics - Simulation and Computation \\
\hline Manuscript ID: & LSSP-2010-0233.R1 \\
\hline Manuscript Type: & Original Paper \\
\hline $\begin{array}{r}\text { Date Submitted by the } \\
\text { Author: }\end{array}$ & 23-Nov-2010 \\
\hline Complete List of Authors: & $\begin{array}{l}\text { Gomes, M. Ivette; Faculdade de Ciências de Lisboa, DEIO } \\
\text { Henriques-Rodrigues, Lígia; Instituto Politécnico de Tomar } \\
\text { Miranda, Cristina; ISCA, Universidade de Aveiro }\end{array}$ \\
\hline Keywords: & $\begin{array}{l}\text { Statistics of extremes, extreme value index, semi-parametric } \\
\text { location/scale invariant estimation, adaptive choice, heuristics, bias } \\
\text { reduction }\end{array}$ \\
\hline Abstract: & $\begin{array}{l}\text { In this paper, we deal with semi-parametric corrected-bias } \\
\text { estimation of a positive extreme value index (EVI). Then, the } \\
\text { classical EVI-estimators are the Hill estimators, based on any } \\
\text { intermediate number k of top order statistics. But these EVI- } \\
\text { estimators are not location-invariant, contrarily to the peaks over } \\
\text { random threshold (PORT)-Hill estimators, which depend on an extra } \\
\text { tuning parameter q. On the basis of second-order minimum- } \\
\text { variance reduced-bias (MVRB) EVI-estimators, we shall here } \\
\text { consider PORT-MVRB EVI-estimators, and propose the use of a } \\
\text { heuristic algorithm, for the adaptive choice of } k \text { and q. Applications } \\
\text { in the fields of insurance and finance will be provided. }\end{array}$ \\
\hline \multicolumn{2}{|c|}{$\begin{array}{l}\text { Note: The following files were submitted by the author for peer review, but cannot be converted } \\
\text { to PDF. You must view these files (e.g. movies) online. }\end{array}$} \\
\hline AlgoI_CSSC_R1.tex & \\
\hline
\end{tabular}




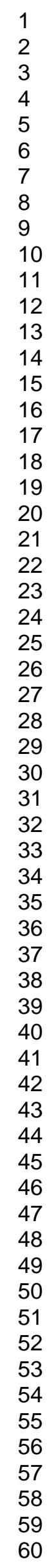

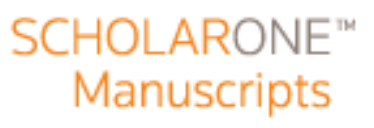

0

12

13

16

17

18

19

20

22

23

25

26

28

29

32

33

34

35

36

39

40

41

42

44

45

46

47

48

50

51 


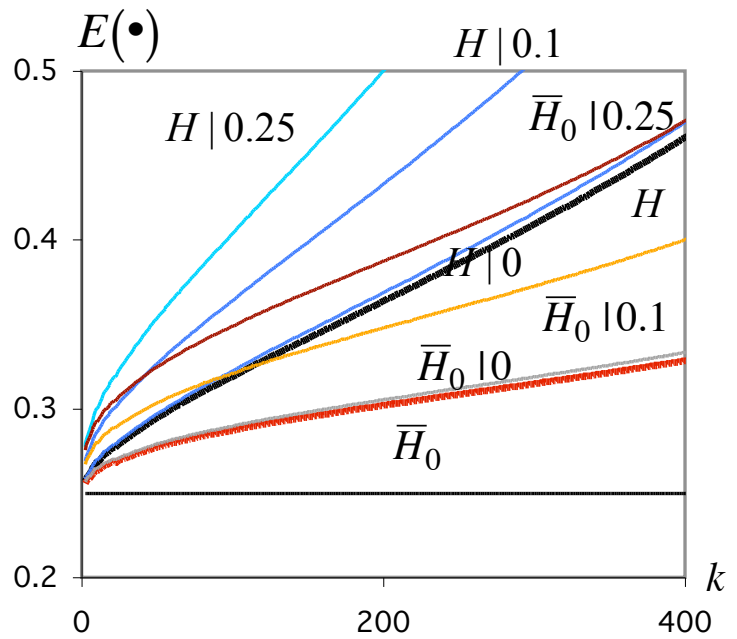




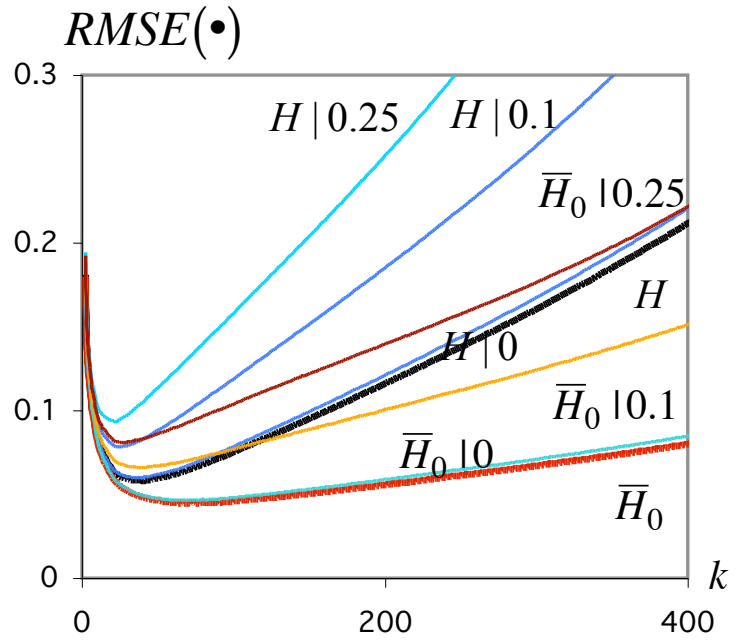




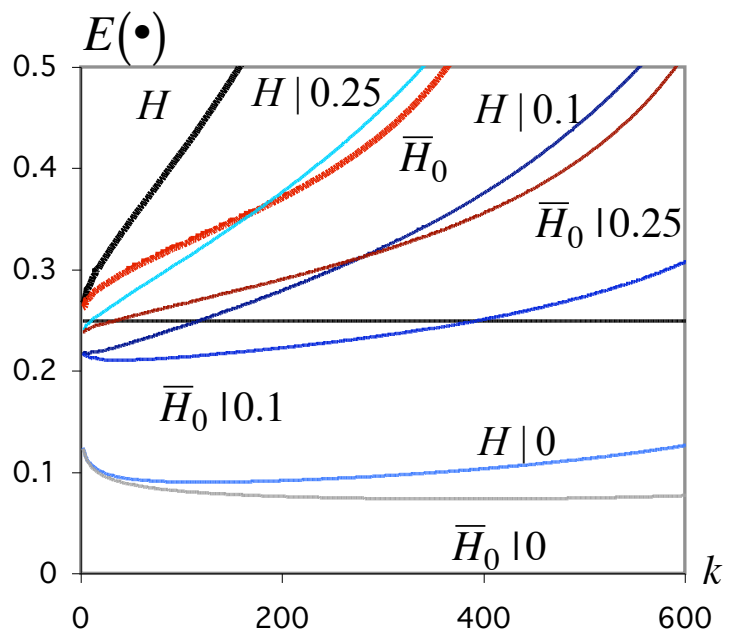




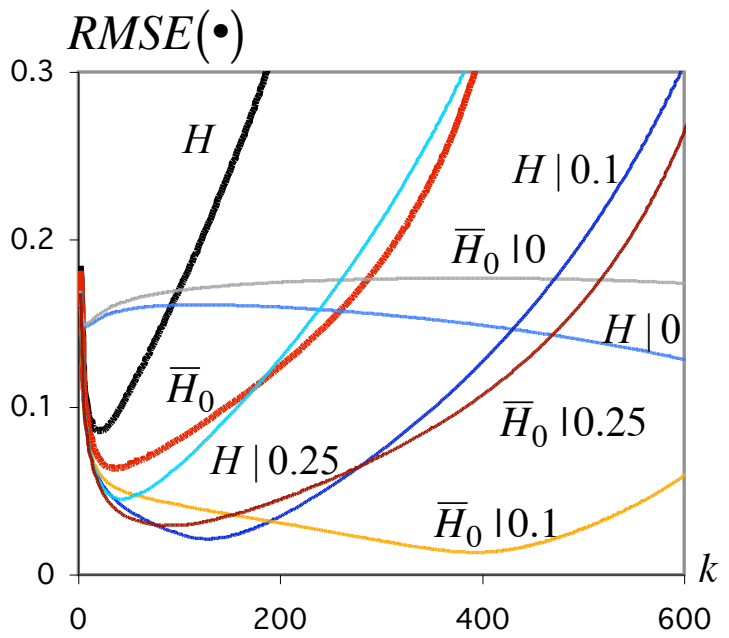




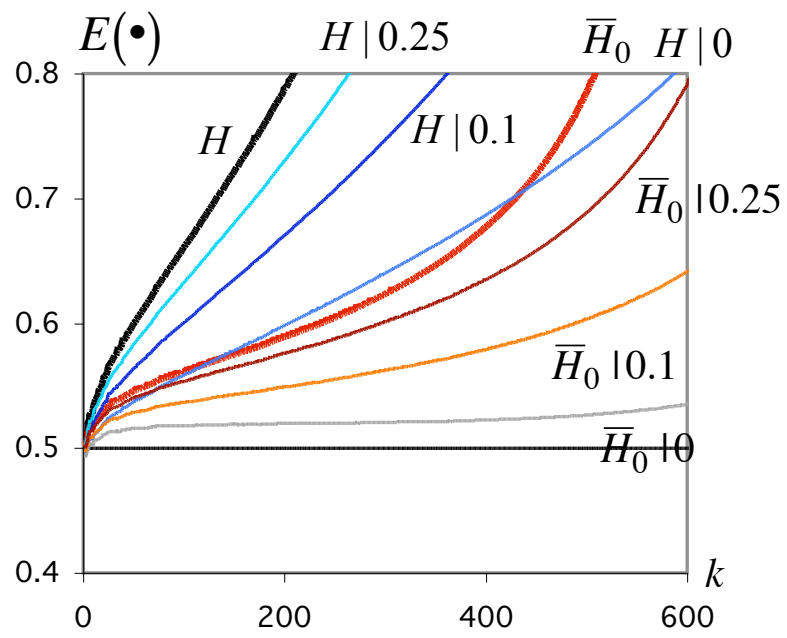




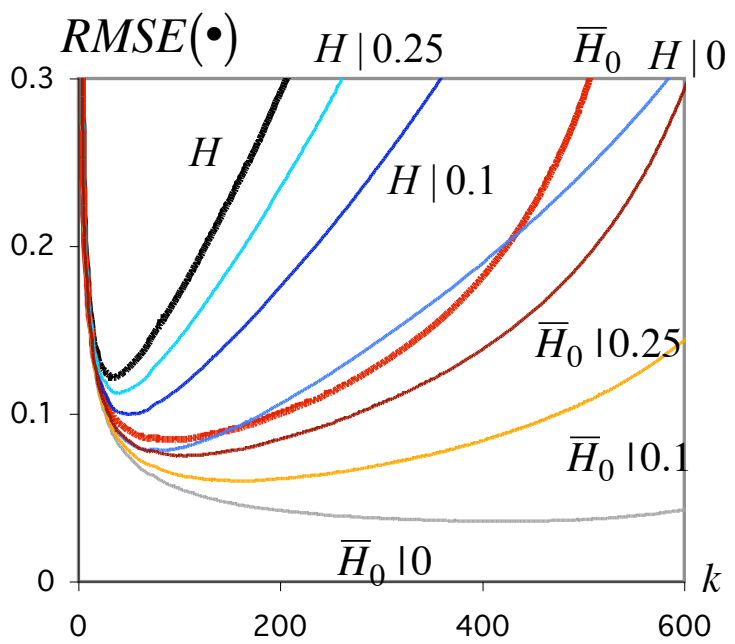


$R E F F_{0}^{\circ}$

1.5

$$
\bar{H}_{0} \mid 0
$$

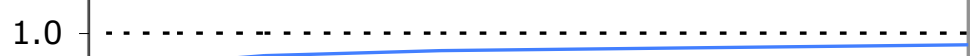

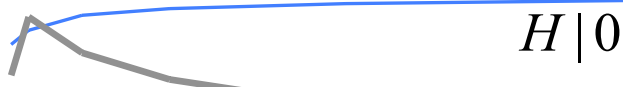

$\bar{H}_{0} \mid 0.25$

$\bar{H}_{0} \mid 0.1$

\section{$H \mid 0.1$}

\section{$H \mid 0.25$}

0.5 


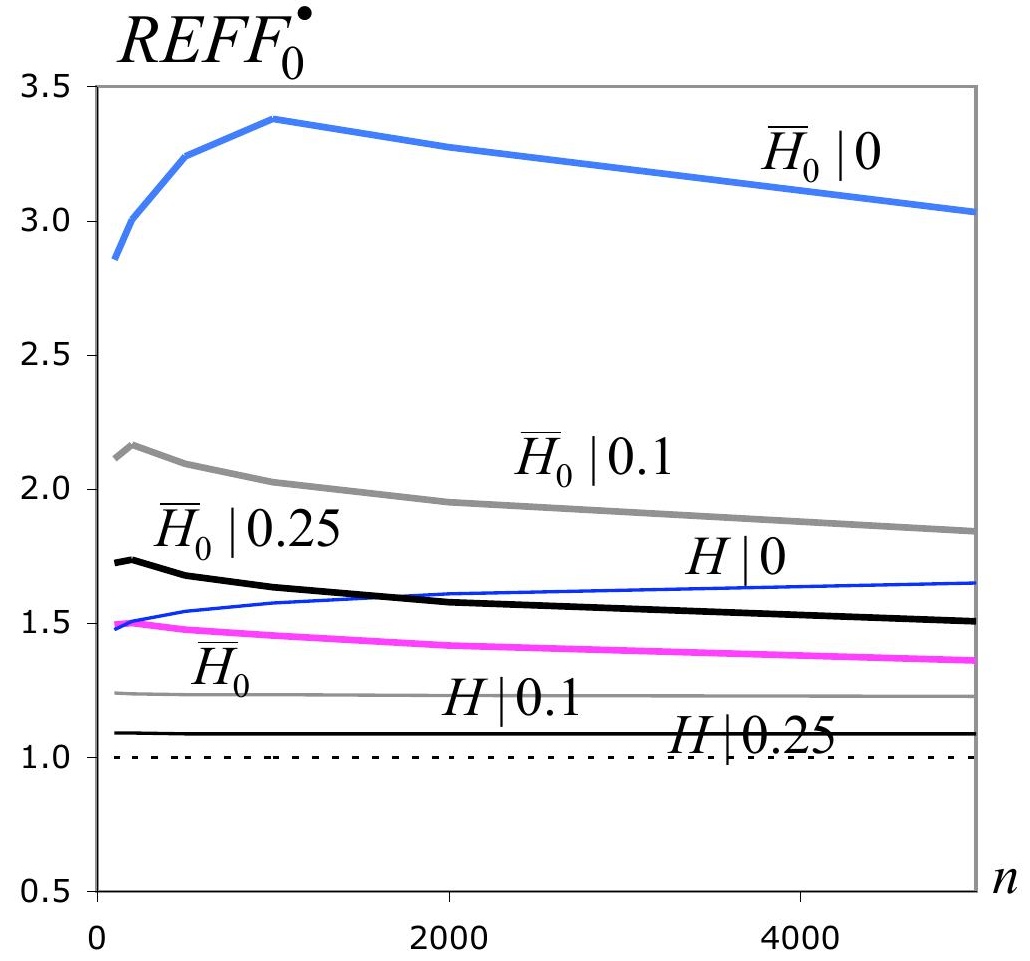




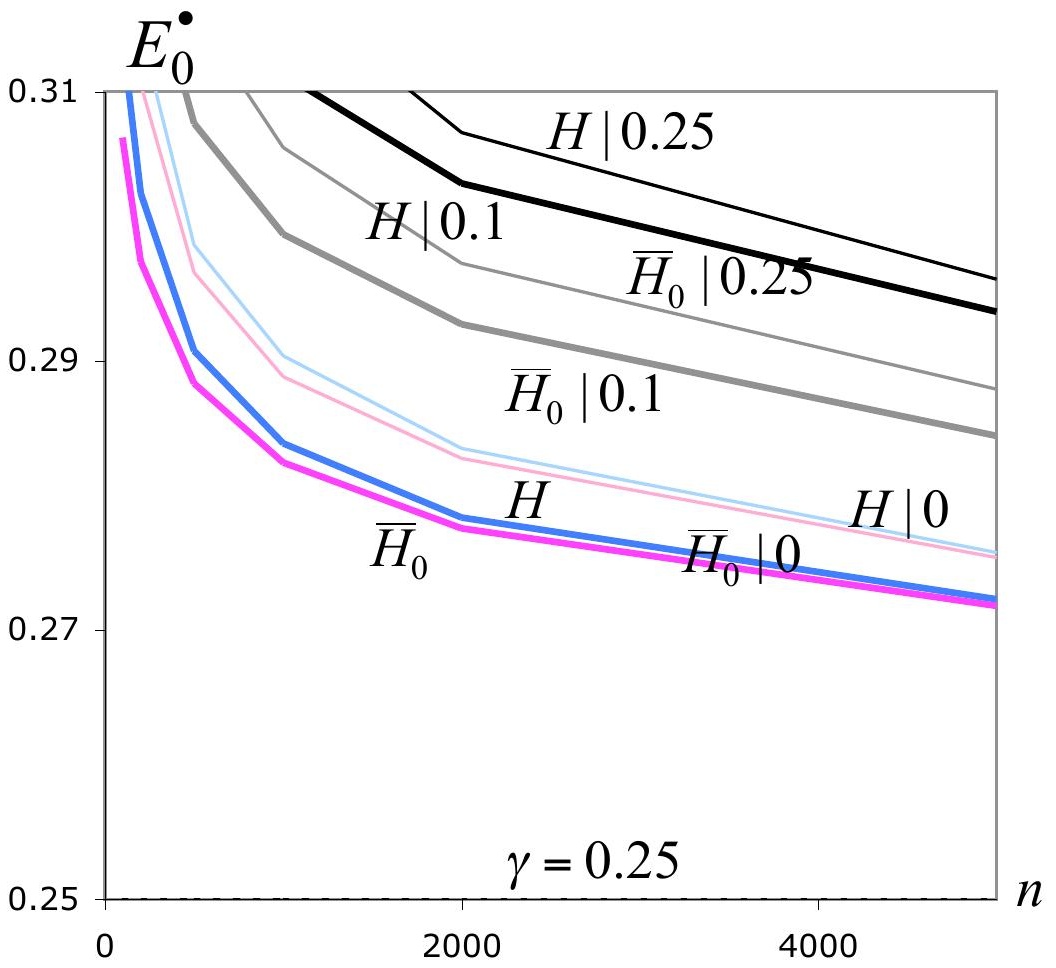




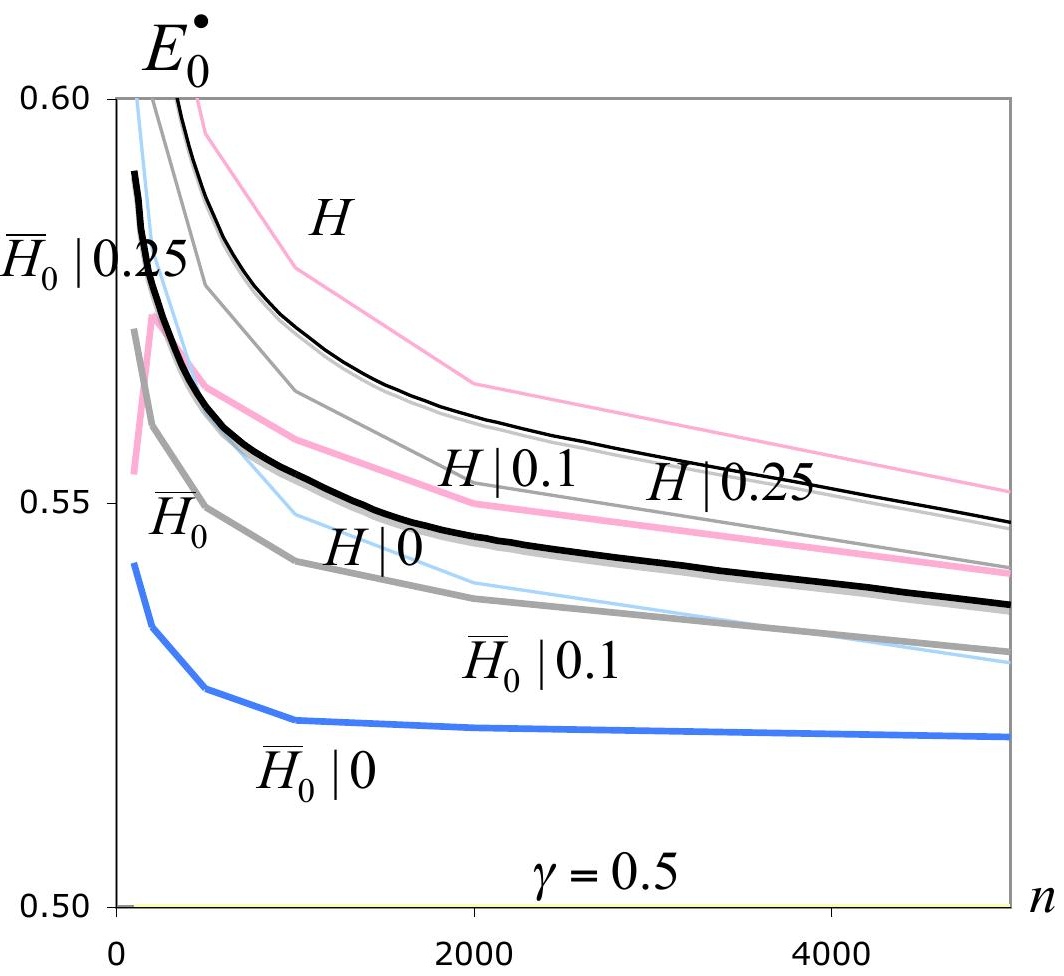




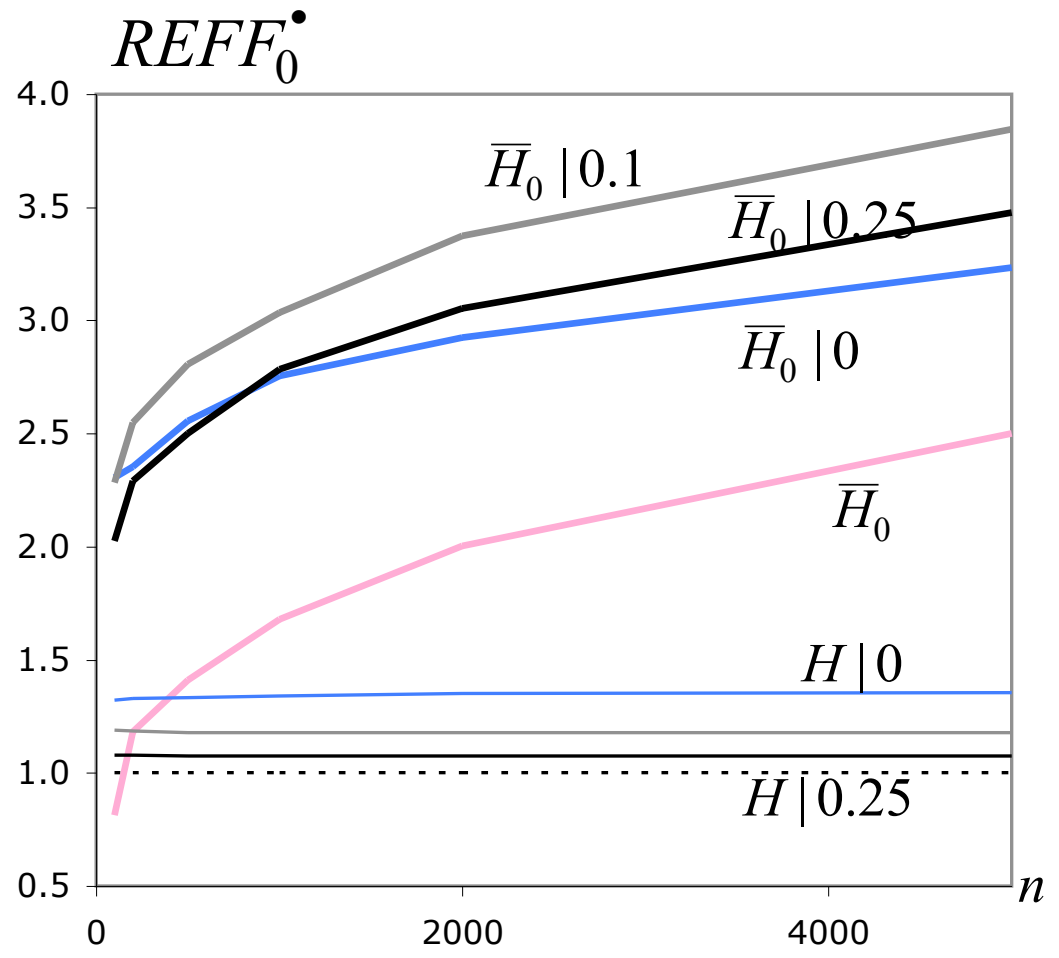




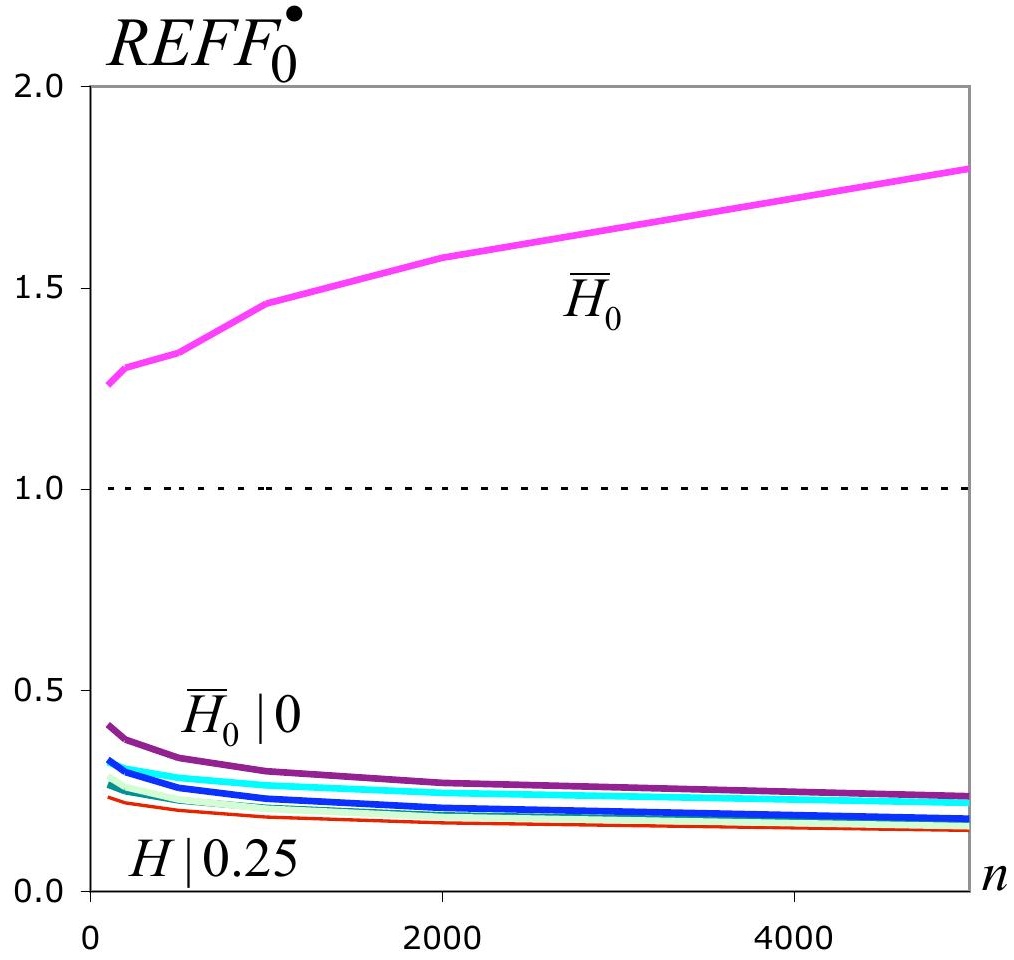




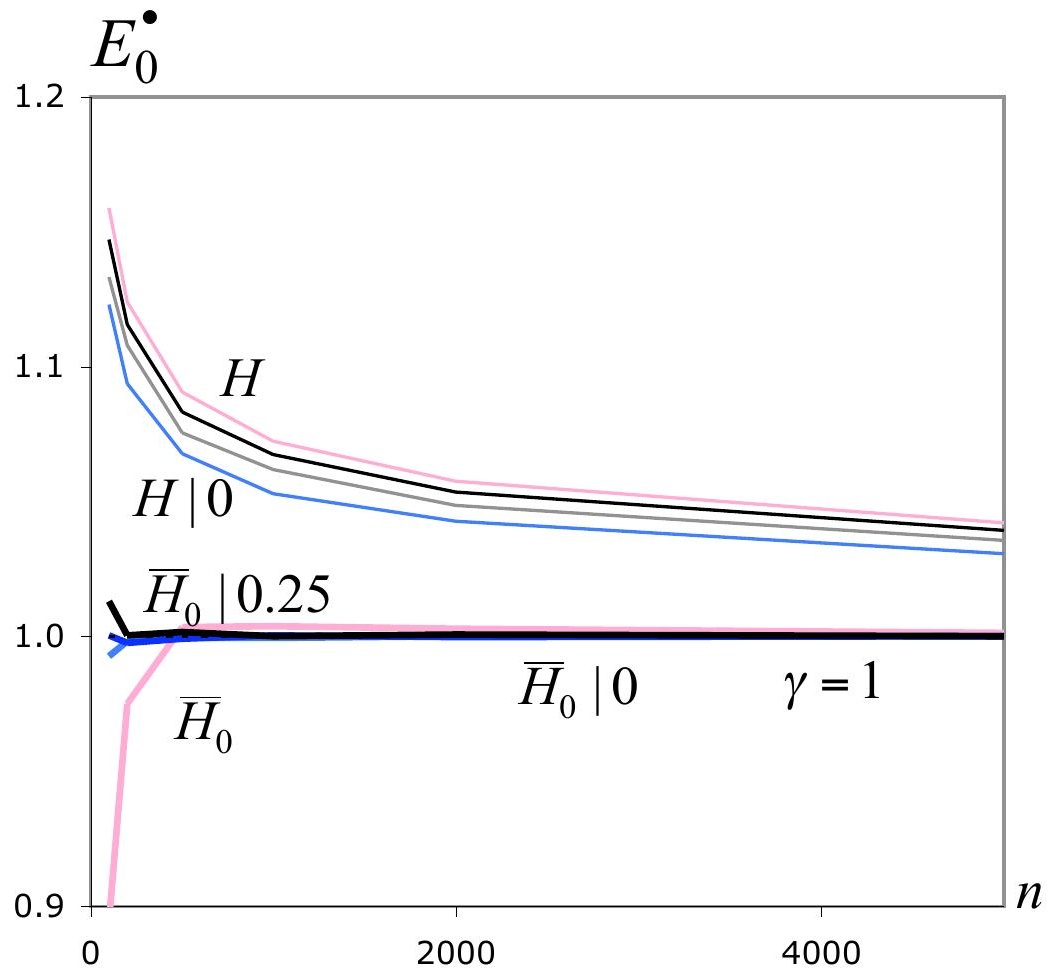




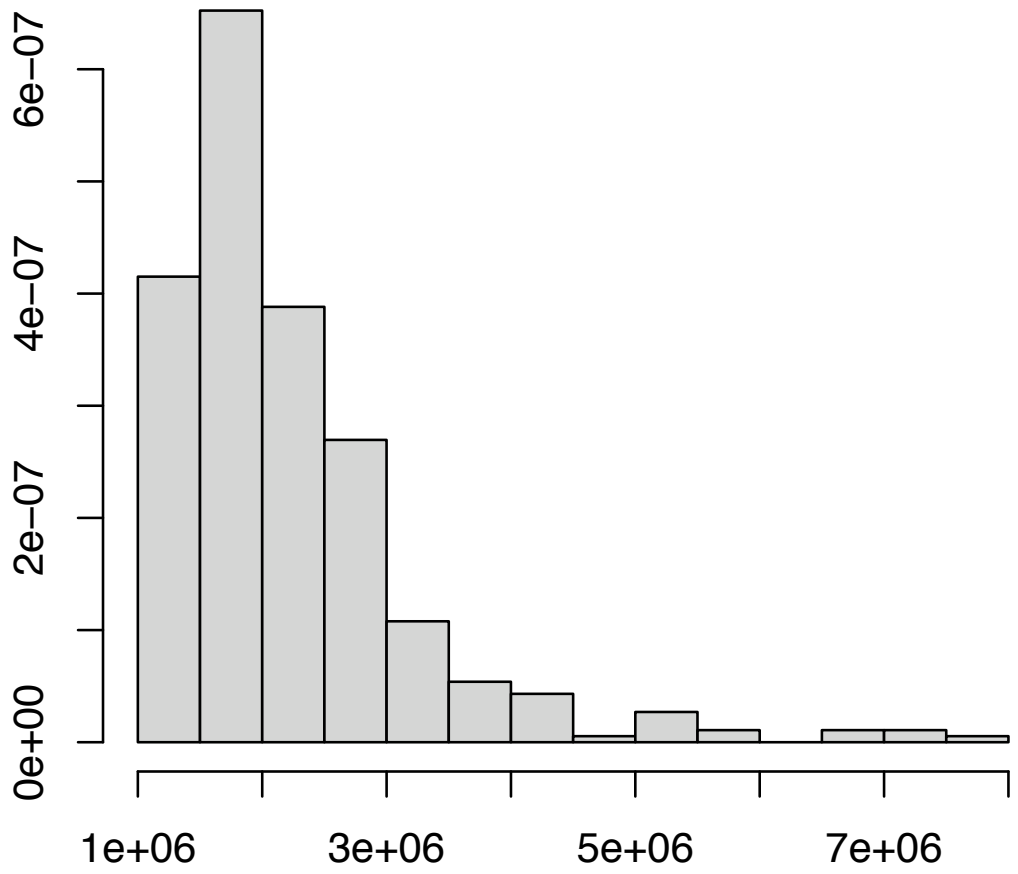

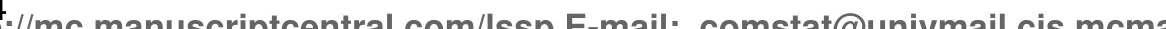




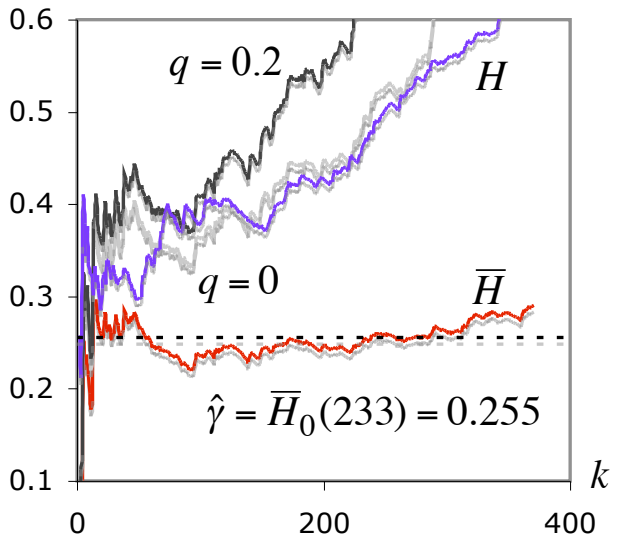




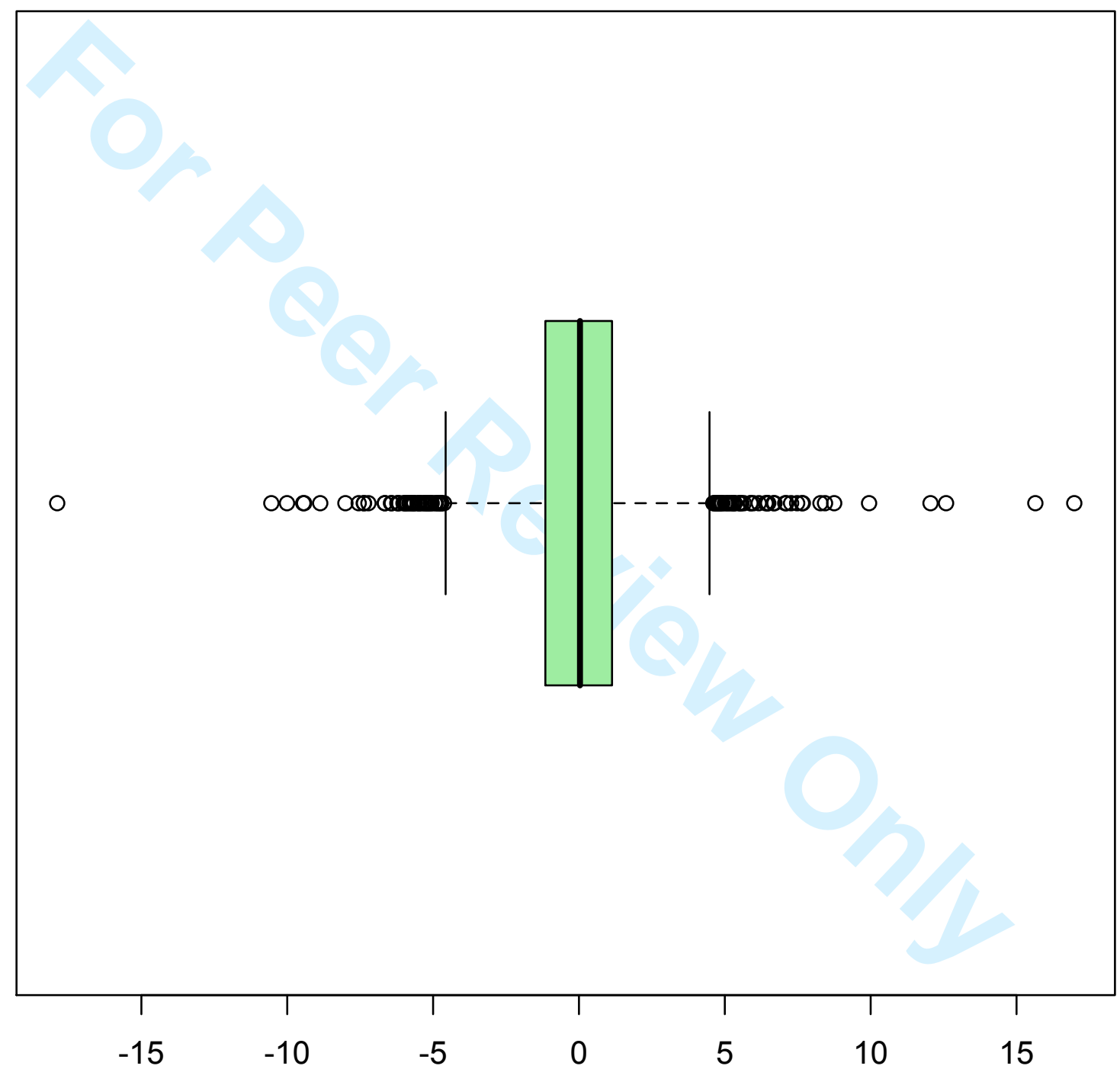




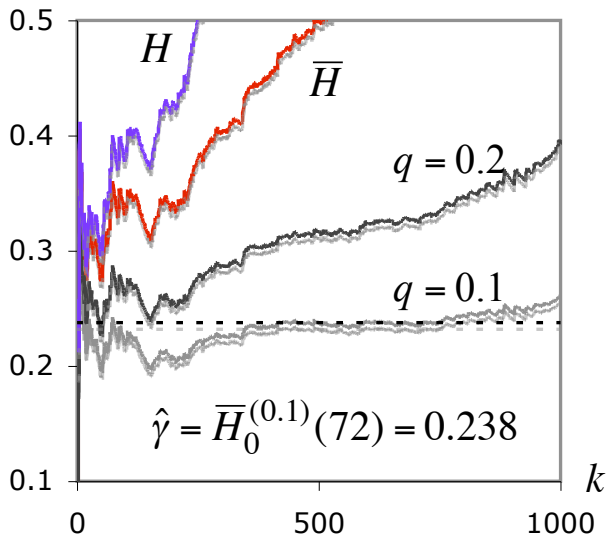




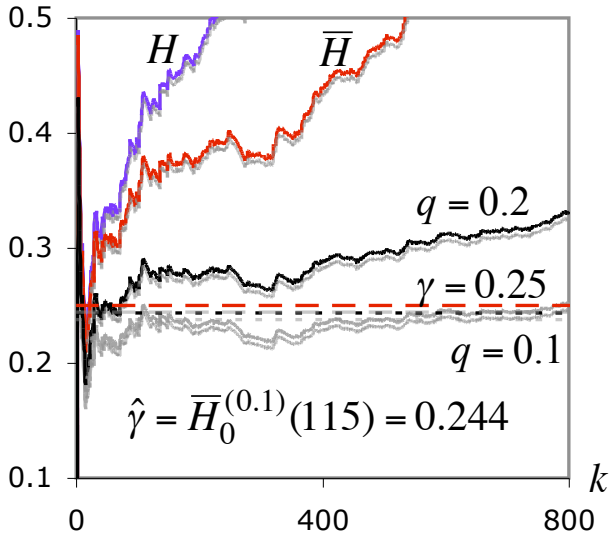




\title{
Reduced-Bias Location-Invariant Extreme Value Index Estimation: a Simulation Study*
}

\author{
M. Ivette Gomes \\ Universidade de Lisboa, FCUL, DEIO and CEAUL \\ Lígia Henriques-Rodrigues M. Cristina Miranda \\ Instituto Politécnico de Tomar, and CEAUL ISCA, Universidade de Aveiro, and CEAUL
}

November 22, 2010

\begin{abstract}
In this paper, we deal with semi-parametric corrected-bias estimation of a positive extreme value index (EVI), the primary parameter in statistics of extremes. Under such a context, the classical EVI-estimators are the Hill estimators, based on any intermediate number $k$ of top order statistics. But these EVI-estimators are not location-invariant, contrarily to the PORT-Hill estimators, which depend on an extra tuning parameter $q$, with $0 \leq q<1$, and where PORT stands for peaks over random threshold. On the basis of second-order minimum-variance reduced-bias (MVRB) EVI-estimators, we shall here consider PORT-MVRB EVI-estimators. Due to the stability on $k$ of the MVRB EVI-estimates, we propose the use of a heuristic algorithm, for the adaptive choice of $k$ and $q$, based on the bias pattern of the estimators as a function of $k$. Applications in the fields of insurance and finance will be provided.
\end{abstract}

${ }^{*}$ Research partially supported by FCT / OE and PTDC / FEDER. 
Keywords. Statistics of extremes; extreme value index; semi-parametric location/scale invariant estimation; adaptive choice; heuristics; bias reduction.

\section{Introduction and preliminaries}

Given an underlying distribution function (d.f.) $F$, and with the notation $F^{\leftarrow}(y):=\inf \{x: F(x) \geq y\}$ for the generalized inverse function of $F$, let us denote $U(t):=F^{\leftarrow}(1-1 / t), t \geq 1$, the associated reciprocal quantile function. As usual, the notation $R V_{\alpha}$ will be used for regularly-varying functions with an index of regular variation equal to $\alpha$, i.e., positive measurable functions $g(\cdot)$ such that for any $x>0, g(t x) / g(t) \rightarrow x^{\alpha}$, as $t \rightarrow \infty$. Under a semi-parametric framework, we deal with the estimation of a positive extreme value index (EVI) $\gamma$, the primary parameter in statistics of extremes, i.e. we shall consider parents such that

$$
U \in R V_{\gamma} \Longleftrightarrow \bar{F}:=1-F \in R V_{-1 / \gamma},
$$

the usually called heavy-tailed parents, quite common in the most diversified areas of application, like finance, insurance, bibliometrics and biology. We are then working in $\mathcal{D}_{\mathcal{M}}^{+} \equiv \mathcal{D}_{\mathcal{M}}\left(E V_{\gamma}\right)_{\gamma>0}$, the domain of attraction for maxima of $E V_{\gamma}, \gamma>0$, with $E V_{\gamma}$ denoting the general extreme value $(\mathrm{EV})$ d.f., given by

$$
E V_{\gamma}(x)= \begin{cases}\exp \left(-(1+\gamma x)^{-1 / \gamma}\right), 1+\gamma x>0 & \text { if } \gamma \neq 0 \\ \exp (-\exp (-x)), x \in \mathbb{R} & \text { if } \gamma=0\end{cases}
$$

For these heavy-tailed parents, given a sample $\underline{\mathbf{X}}_{n}=\left(X_{1}, \ldots, X_{n}\right)$ and the associated sample of ascending order statistics (o.s.'s), $\left(X_{1: n} \leq \cdots \leq X_{n: n}\right)$, the classical EVI-estimators are Hill estimators (Hill, 1975), here denoted $H \equiv H(k), k=1,2, \ldots, n-1$, and given by

$$
H(k) \equiv H\left(k ; \underline{\mathbf{X}}_{n}\right):=\frac{1}{k} \sum_{i=1}^{k}\left\{\ln X_{n-i+1: n}-\ln X_{n-k: n}\right\},
$$


the average of the $k$ log-excesses over a high random threshold $X_{n-k: n}$. Consistency of the estimators in (2) is achieved if $X_{n-k: n}$ is an intermediate o.s., i.e., if

$$
k=k_{n} \rightarrow \infty \quad \text { and } \quad k / n \rightarrow 0, \text { as } n \rightarrow \infty
$$

The Hill estimators, in (2), are scale-invariant, but not location-invariant, as often desired, and this contrarily to the PORT-Hill estimators, introduced in Araújo Santos et al. (2006) and further studied in Gomes et al. (2008a), with PORT standing for peaks over random thresholds. The class of PORT-Hill estimators is based on a sample of excesses over a random threshold $X_{n_{q}: n}$, i.e., it is based on

$$
\underline{\mathbf{X}}_{n}^{(q)}:=\left(X_{n: n}-X_{n_{q}: n}, \ldots, X_{n_{q}+1: n}-X_{n_{q}: n}\right), \quad \text { with } \quad n_{q}:=[n q]+1,
$$

where $[x]$ denotes, as usual, the integer part of $x$. We can generally have $0<q<1$, for d.f.'s with finite or infinite left endpoint $x_{F}:=\inf \{x: F(x)>0\}$ (the random threshold is an empirical quantile), and $q=0$, for d.f.'s with finite left endpoint $x_{F}$ (the random threshold is the minimum). These new classes of EVI-estimators are the so-called PORTHill estimators, denoted $H \mid q \equiv H^{(q)} \equiv H^{(q)}(k)$ and given by

$$
H^{(q)}(k):=H\left(k ; \underline{\mathbf{X}}_{n}^{(q)}\right)=\frac{1}{k} \sum_{i=1}^{k} \ln \frac{X_{n-i+1: n}-X_{n_{q}: n}}{X_{n-k: n}-X_{n_{q}: n}}, \quad 0 \leq q<1, \quad k<n-n_{q},
$$

i.e., they have the same functional form of the Hill estimator in (2), but with the original sample $\underline{\mathbf{X}}_{n}=\left(X_{1}, \ldots, X_{n}\right)$ replaced by the sample of excesses $\underline{\mathbf{X}}_{n}^{(q)}$ in (4). These estimators are now invariant for both changes of scale and location in the data, and depend on the tuning parameter $q$, that provides a highly flexible class of EVI-estimators, which may even compare favorably with the second-order minimum-variance reduced-bias (MVRB) EVI-estimators described in the following, provided that we adequately choose the tuning parameter $q$. Indeed, and due to the high bias of the Hill estimator, in (2), for moderate up to large $k$, several authors have been dealing with bias reduction in the field of extremes, working usually in a slightly more restrict class than $\mathcal{D}_{\mathcal{M}}^{+}$, the class of models $U(\cdot)$ such that

$$
U(t)=C t^{\gamma}\left(1+A(t) / \rho+o\left(t^{\rho}\right)\right), \quad A(t)=\gamma \beta t^{\rho}
$$


as $t \rightarrow \infty$, where $C, \gamma>0, \rho<0$ and $\beta \neq 0$. This means that the slowly varying function $L(t)$ in $U(t)=t^{\gamma} L(t)$ is assumed to behave asymptotically as a constant $C$. Note that to assume (6) is equivalent to saying that we can choose $A(t)=\gamma \beta t^{\rho}, \rho<0$, in the more general second-order condition

$$
\lim _{t \rightarrow \infty} \frac{\ln U(t x)-\ln U(t)-\gamma \ln x}{A(t)}=\frac{x^{\rho}-1}{\rho} .
$$

Indeed, the Hill estimator reveals usually a high asymptotic bias, i.e., as $n \rightarrow \infty$, and if (3) and (7) hold, $\sqrt{k}(H(k)-\gamma)$ is asymptotically normal with variance $\gamma^{2}$ and a non-null mean value, equal to $\lambda /(1-\rho)$, whenever $\sqrt{k} A(n / k) \rightarrow \lambda \neq 0$, finite, with $A(\cdot)$ the function in (7). This non-null asymptotic bias, together with a rate of convergence of the order of $1 / \sqrt{k}$, leads to sample paths with a high variance for small $k$, a high bias for large $k$, and a very sharp mean squared error $(M S E)$ pattern, as a function of $k$. A simple class of second-order MVRB EVI-estimators is the one in Caeiro et al. (2005), used for a semi-parameteric estimation of $\ln V a R_{p}$ in Gomes and Pestana (2007). This class, here denoted $\bar{H} \equiv \bar{H}(k)$, depends upon the estimation of the second-order parameters $(\beta, \rho)$ in (6). Its functional form is

$$
\bar{H}(k) \equiv \bar{H}\left(k ; \underline{\mathbf{X}}_{n}\right) \equiv \bar{H}_{\hat{\beta}, \hat{\rho}}(k):=H(k)\left(1-\hat{\beta}(n / k)^{\hat{\rho}} /(1-\hat{\rho})\right),
$$

with $H(k)$ the Hill estimator in $(2)$, and where $(\hat{\beta}, \hat{\rho})$ needs to be an adequate consistent estimator of $(\beta, \rho)$. Then, under the same conditions as before, i.e., if (3) holds and $\sqrt{k} A(n / k) \rightarrow \lambda \neq 0$, finite, $\sqrt{k}(\bar{H}(k)-\gamma)$ is asymptotically normal with variance also equal to $\gamma^{2}$ but with a null mean value. Indeed, it follows from the results of de Haan and Peng (1998) that for models in (6), and with $H(k)$ given in (2),

$$
\sqrt{k}(H(k)-\gamma) \stackrel{d}{=} \operatorname{Normal}\left(0, \gamma^{2}\right)+\frac{\gamma \beta \sqrt{k}(n / k)^{\rho}}{1-\rho}\left(1+o_{p}(1)\right)
$$

where the bias $\gamma \beta \sqrt{k}(n / k)^{\rho} /(1-\rho)$ can be very large, moderate or small (i.e. go to infinity, constant or zero) as $n \rightarrow \infty$. Also, from the results in Caeiro et al. (2005), we know that it is possible to adequately estimate the second-order parameters $\beta$ and $\rho$, so that we get 


$$
\sqrt{k}(\bar{H}(k)-\gamma) \stackrel{d}{=} \operatorname{Normal}\left(0, \gamma^{2}\right)+o_{p}\left(\sqrt{k}(n / k)^{\rho}\right),
$$

for $\bar{H}(k)$ in $(8)$, i.e. $\bar{H}(k)$ outperforms $H(k)$ for all $k$.

In this paper, we shall consider the new EVI-estimators,

$$
\bar{H}^{(q)}(k):=\bar{H}\left(k ; \underline{\mathbf{X}}_{n}^{(q)}\right)
$$

with the same functional form of the MVRB estimators in (8), but with the original sample $\underline{\mathbf{X}}_{n}$ replaced by the sample of excesses $\underline{\mathbf{X}}_{n}^{(q)}$, in (4). The estimators $\bar{H}^{(q)}(k)$, in (10), will be named PORT-MVRB estimators. They are invariant for both changes of location and scale and, similarly to the relationship between $H$ and $\bar{H}$, it is obvious that for any $q, \bar{H}^{(q)}(k)$ outperforms $H^{(q)}(k)$ for all $k$. A full theoretical study of these PORT-MVRB estimators, with detailed information on the dominant component of bias, is still under investigation, and out of the scope of this paper.

Remark 1. On the basis of (9), the optimal k-value for the EVI-estimation through the Hill estimator in $(2)$, i.e. $k_{0}^{H}:=\arg \min M S E(H(k))$, is thus well approximated by

$$
\underset{k}{\arg \min }\left\{1 / k+\beta^{2}(n / k)^{2 \rho} /(1-\rho)^{2}\right\}=\left((1-\rho)^{2} n^{-2 \rho} /\left(\beta^{2}(-2 \rho)\right)^{1 /(1-2 \rho)}\right) .
$$

Remark 2. With the notation $b_{k, n, \rho}=1+\beta(n / k)^{\rho} /(1-\rho)$, and provided that $\sqrt{k}(n / k)^{\rho} \rightarrow \lambda$, finite, $\sqrt{k}\left\{H(k) / \gamma-b_{k, n, \rho}\right\}$ is approximately $\operatorname{Normal}(0,1)$. We can then get approximate $100(1-\alpha) \%$ confidence intervals $\left(C I^{\prime} s\right)$ for $\gamma$, given by

$$
\left(H(k) /\left(b_{k, n, \rho}+\xi_{1-\alpha / 2} / \sqrt{k}\right), H(k) /\left(b_{k, n, \rho}-\xi_{1-\alpha / 2} / \sqrt{k}\right)\right)
$$

where $\xi_{p}$ denotes the quantile of probability $p$ of a standard normal d.f. If $\lambda=0$, we can replace in (12) the bias summand $\beta(n / k)^{\rho} /(1-\rho)$ by 0 , i.e., we can take $b_{k, n, \rho}=1$.

Remark 3. If we consider a second-order MVRB EVI-estimator, and levels $k$ such that $\sqrt{k}(n / k)^{\rho} \rightarrow \lambda$, finite, we can also easily get even simpler approximate $100(1-\alpha) \% C I^{\prime} s$ 
for $\gamma$. On the basis of the statistic $\bar{H}$ in (8), or even $\bar{H}^{(q)}$ in (10), for adequate values of $q$, and for this same type of levels $k$, we get the following $100(1-\alpha) \%$ approximate $C I$ for $\gamma$,

$$
\left(\bar{H}(k) /\left(1+\xi_{1-\alpha / 2} / \sqrt{k}\right), \bar{H}(k) /\left(1-\xi_{1-\alpha / 2} / \sqrt{k}\right)\right) .
$$

Algorithms for the estimation of $(\beta, \rho)$ are provided in Gomes and Pestana (2007), among others, and such estimation will be only briefly reformulated in Section 2 of this paper. In Section 3, we describe the results associated with a large-scale Monte-Carlo simulation study of the new PORT-MVRB EVI-estimators, in (10). Section 4 is dedicated to a data-driven choice of the tuning parameters $k$ and $q$, inspired in the heuristic choice considered in Figueiredo et al. (2010) for the Value-at-Risk estimation. In Section 4.1, due to the reasonably high stability on $k$ of the MVRB estimates $\bar{H}$, in (8), and $\bar{H}^{(q)}$, in (10), for adequate values of $q$, we provide an algorithm for the choice of $k$, whenever we use $\bar{H}$, in (8), as well as for the choice of $k$ and $q$, whenever we use $\bar{H}^{(q)}$, in (10), as estimators of the EVI. Such algorithm is based on the bias pattern of the estimators as a function of $k$. Finally, in Section 4.2, we provide applications of the adaptive methodology to data in the fields of insurance and finance, as well as to a simulated sample from a Student underlying parent.

\section{Estimation of second-order parameters}

All reduced-bias EVI-estimators, like the ones in (8) and (10), require the estimation of shape and scale second-order parameters, $\beta$ and $\rho$, respectively. Also, the estimation of the optimal sample fraction (OSF) for classical EVI-estimators, like the Hill estimator, in (2), i.e. the sample fraction $k_{0}^{H} / n$, with $k_{0}^{H}$ defined in Remark 1 , depends on the estimation of $\beta$ and $\rho$, in (6). Indeed, on the basis of (11) and with $(\hat{\beta}, \hat{\rho})$ any consistent estimator of $(\beta, \rho)$, we can use

$$
\hat{k}_{0}^{H}:=\left(\frac{(1-\hat{\rho})^{2} n^{-2 \hat{\rho}}}{-2 \hat{\rho} \hat{\beta}^{2}}\right)^{1 /(1-2 \hat{\rho})}
$$

(Hall, 1982). Such an estimation will next be briefly discussed. 
For models in (6), and after taking a decision of working in the region $|\rho| \leq 1$, the region where bias reduction is indeed needed, as well as common in applications to real data, we consider a particular member of the class of estimators introduced in Fraga Alves et al. (2003), parameterized in a tuning real parameter $\tau$, here taken equal to zero, the value suggested in previous papers whenever working in this region of $\rho$-values. Given a sample, $\underline{\mathbf{X}}_{n}$, we shall thus essentially work with $\tau=0$, i.e., with

$$
\hat{\rho}_{0}(k) \equiv \hat{\rho}_{0}\left(k ; \underline{\mathbf{X}}_{n}\right):=\min \left(0, \frac{3\left(T_{n}^{(0)}\left(k ; \underline{\mathbf{X}}_{n}\right)-1\right)}{T_{n}^{(0)}\left(k ; \underline{\mathbf{X}}_{n}\right)-3}\right),
$$

dependent on the statistics

$$
T_{n}^{(0)}\left(k ; \underline{\mathbf{X}}_{n}\right):=\frac{\ln \left(M_{n}^{(1)}\left(k ; \underline{\mathbf{X}}_{n}\right)\right)-\frac{1}{2} \ln \left(M_{n}^{(2)}\left(k ; \underline{\mathbf{X}}_{n}\right) / 2\right)}{\frac{1}{2} \ln \left(M_{n}^{(2)}\left(k ; \underline{\mathbf{X}}_{n}\right) / 2\right)-\frac{1}{3} \ln \left(M_{n}^{(3)}\left(k ; \underline{\mathbf{X}}_{n}\right) / 6\right)},
$$

where

$$
M_{n}^{(j)}(k):=\frac{1}{k} \sum_{i=1}^{k}\left\{\ln X_{n-i+1: n}-\ln X_{n-k: n}\right\}^{j}, \quad j=1,2,3 .
$$

In the simulations we shall however also consider the estimator in Fraga Alves et al. (2003), associated with $\tau=1$, i.e.

$$
\hat{\rho}_{1}(k) \equiv \hat{\rho}_{1}\left(k ; \underline{\mathbf{X}}_{n}\right):=\min \left(0, \frac{3\left(T_{n}^{(1)}\left(k ; \underline{\mathbf{X}}_{n}\right)-1\right)}{T_{n}^{(1)}\left(k ; \underline{\mathbf{X}}_{n}\right)-3}\right),
$$

where, with $M_{n}^{(j)}(k)$ given in $(15)$,

$$
T_{n}^{(1)}\left(k ; \underline{\mathbf{X}}_{n}\right):=\frac{\left(M_{n}^{(1)}\left(k ; \underline{\mathbf{X}}_{n}\right)\right)-\left(M_{n}^{(2)}\left(k ; \underline{\mathbf{X}}_{n}\right) / 2\right)^{1 / 2}}{\left(M_{n}^{(2)}\left(k ; \underline{\mathbf{X}}_{n}\right) / 2\right)^{1 / 2}-\left(M_{n}^{(3)}\left(k ; \underline{\mathbf{X}}_{n}\right) / 6\right)^{1 / 3}}
$$

Distributional properties of the estimators in (14) and (16) can be found in Fraga Alves et al. (2003). Consistency is achieved in the class of models in (6), for intermediate $k$-values, i.e., $k$-values such that (3) holds, and also such that $\sqrt{k} A(n / k) \rightarrow \infty$, as $n \rightarrow \infty$. As already suggested in previous papers, we have here decided for the computation of $\hat{\rho}_{\tau}(k)$, $\tau=0,1$, at $k=k_{1}$, given by

$$
k_{1}=\left[n^{1-\epsilon}\right], \quad \epsilon=0.001
$$


the threshold used in Caeiro et al. (2005) and Gomes and Pestana (2007). With such a choice of $k_{1}$, and whenever $\sqrt{k_{1}} A\left(n / k_{1}\right) \rightarrow \infty$, we get $\hat{\rho}-\rho:=\hat{\rho}_{\tau}\left(k_{1}\right)-\rho=o_{p}(1 / \ln n)$, a condition needed, in order not to have any increase in the asymptotic variance of the bias-corrected Hill estimators in equations (8) and (10). Note that with the choice of $k_{1}$ in (17), we get $\sqrt{k_{1}} A\left(n / k_{1}\right) \rightarrow \infty$ if and only if $\rho>1 / 2-1 /(2 \epsilon)=-499.5$, an irrelevant restriction, from a practical point of view. Interesting alternative classes of $\rho$-estimators have recently been introduced in Goegebeur et al. $(2008,2010)$ and Ciuperca and Mercadier (2010).

For the estimation of the scale second-order parameter $\beta$, in (6), and again on the basis of a sample $\underline{\mathbf{X}}_{n}$, we shall here consider

$$
\hat{\beta}_{\hat{\rho}}(k) \equiv \hat{\beta}_{\hat{\rho}}\left(k ; \underline{\mathbf{X}}_{n}\right):=\left(\frac{k}{n}\right)^{\hat{\rho}} \frac{d_{\hat{\rho}}(k) D_{0}(k)-D_{\hat{\rho}}(k)}{d_{\hat{\rho}}(k) D_{\hat{\rho}}(k)-D_{2 \hat{\rho}}(k)},
$$

dependent on the estimator $\hat{\rho}=\hat{\rho}_{\tau}\left(k_{1} ; \underline{\mathbf{X}}_{n}\right), \tau=0$ or 1 , suggested before and where, for any $\alpha \leq 0$,

$$
d_{\alpha}(k):=\frac{1}{k} \sum_{i=1}^{k}\left(\frac{i}{k}\right)^{-\alpha} \quad \text { and } \quad D_{\alpha}(k):=\frac{1}{k} \sum_{i=1}^{k}\left(\frac{i}{k}\right)^{-\alpha} U_{i}, \quad U_{i}:=i\left(\ln \frac{X_{n-i+1: n}}{X_{n-i: n}}\right),
$$

with $U_{i}, 1 \leq i \leq k$, the scaled log-spacings associated with $\underline{\mathbf{X}}_{n}$. Details on the distributional behaviour of the estimator in (18) can be found in Gomes and Martins (2002) and more recently in Gomes et al. (2008b) and Caeiro et al. (2009). Consistency is achieved for models in (6), $k$ values such that (3) holds and $\sqrt{k} A(n / k) \rightarrow \infty$, as $n \rightarrow \infty$, and estimators $\hat{\rho}$ of $\rho$ such that $\hat{\rho}-\rho=o_{p}(1 / \ln n)$. Alternative estimators of $\beta$ can be found in Caeiro and Gomes (2006) and Gomes et al. (2010).

\section{Remarks}

- As mentioned before, bias reduction is really needed when $|\rho| \leq 1$, a common situation in practice. Hence the fundamental role played by the tuning parameter $\tau=0$, and the estimators in (14).

- In the simulation study, the EVI-estimators in (8) and (10) will be denoted $\bar{H}_{0}$ and 
$\bar{H}_{0}^{(q)}$, respectively, to enhance the choice $\tau=0$ in the class of $\rho$-estimators in Fraga Alves et al. (2003). For $\tau=1$, we shall use the obvious notation $\bar{H}_{1}$ and $\bar{H}_{1}^{(q)}$.

\section{Finite sample behaviour: a Monte-Carlo simulation}

In this section, for $q=0,0.1$ and 0.25 , we are interested in the finite-sample behaviour of the PORT-MVRB EVI-estimators, $\bar{H}^{(q)}(k)$, in (10), comparatively with the classical Hill EVI-estimators, $H(k)$, in (2) and the MVRB EVI-estimators $\bar{H}(k)$, in (8). We have performed a large-scale multi-sample simulation with size $5000 \times 20$, i.e., 20 replicates with 5000 runs each. For details on multi-sample simulation refer to Gomes and Oliveira (2001). The patterns of mean values (E) and root mean squared errors (RMSE) are based on the first replicate. As an illustration, we shall present in this article the results associated with the following underlying parents, all with a shape second-order parameter equal to -0.5 :

I. the Burr model, with d.f. $F(x)=1-\left(1+x^{-\rho / \gamma}\right)^{1 / \rho}, x \geq 0, \gamma>0$, with $\gamma=0.25$ and $\rho=-0.5$

II. the Student's $t_{\nu}$-model with $\nu$ degrees of freedom, with a probability density function

$$
f_{t_{\nu}}(t)=\Gamma((\nu+1) / 2)\left[1+t^{2} / \nu\right]^{-(\nu+1) / 2} /(\sqrt{\pi \nu} \Gamma(\nu / 2)), t \in \mathbb{R} \quad(\nu>0),
$$

with $\nu=4$ degrees of freedom $(\gamma=0.25$ and $\rho=-0.5)$;

III. the general $E V_{\gamma}$ model, in (1), with $\gamma=0.5(\rho=-0.5)$.

\subsection{Mean values and mean squared errors paterns}

In Figures 1, 2 and 3, for the models in I., II. and III., respectively, we show the simulated patterns of mean value, $\mathrm{E}(\bullet)$, and root mean squared error, $\operatorname{RMSE}(\bullet)$, of the EVI-estimators $H(k), H^{(q)}(k), \bar{H}_{0}(k)$ and $\bar{H}_{0}^{(q)}(k)$, with $H, H^{(q)}, \bar{H}$ and $\bar{H}^{(q)}$ defined in (2), (5), (8) and (10), respectively. For the sake of simplicity, we denote these estimates by $H, H \mid q, \bar{H}_{0}$ and $\bar{H}_{0} \mid q$, respectively. 

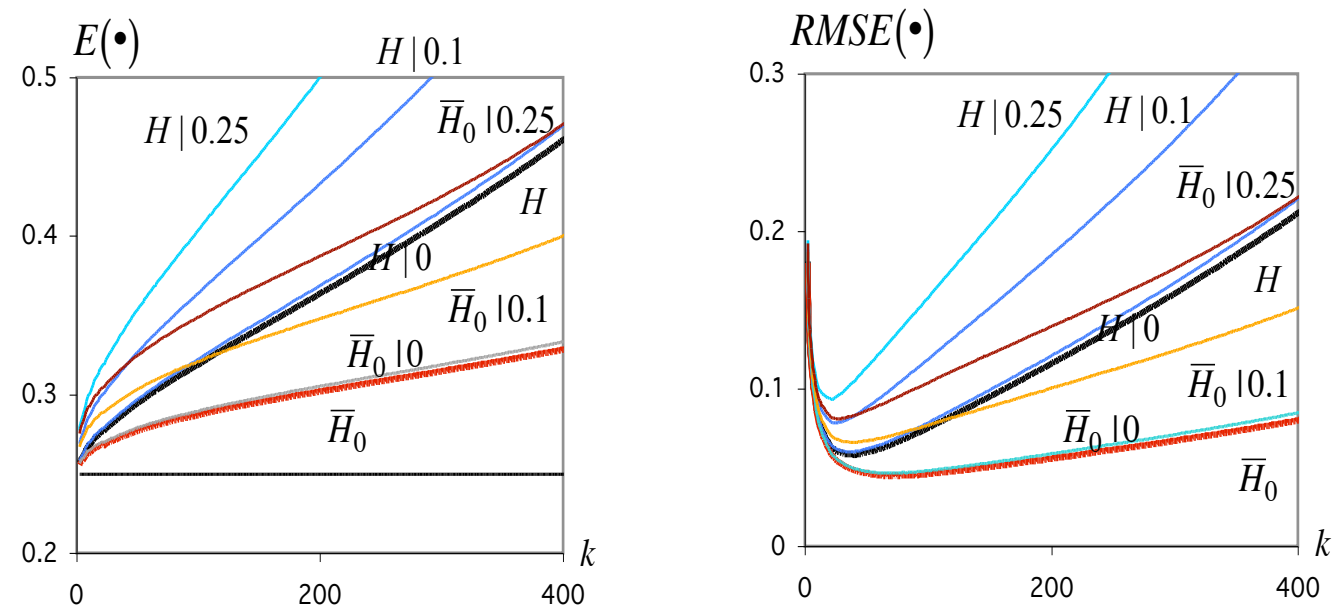

Figure 1: Patterns of mean values (left) and root mean squared errors (right), as functions of $k$, for an underlying Burr parent with $(\gamma, \rho)=(0.25,-0.5)$.

These parents were chosen just to illustrate the fact that:

- The PORT-MVRB EVI-estimators can be not able to improve the performance of $\bar{H}_{0}$, as happens also with the PORT-Hill estimators when compared with the Hill estimator $H$ (see Figure 1, associated with the Burr model). Indeed, this hapens for all models with a left endpoint greater than or equal to zero.

- The PORT-Hill estimators can outperform the MVRB-estimator $\bar{H}_{0}$ (see Figure 2, associated with a Sudent $t_{4}$ underlying parent). For this type of models, with a left endpoint equal to infinity, the value $q=0$ should be discarded due to inconsistency (see the patterns of $H \mid 0$ and $\bar{H}_{0} \mid 0$ in Figure 2, and Gomes et al., 2008a, for further details on the subject).

- We can often find a value of $q$ that provides the best estimator of $\gamma$, regarding for instance minimum MSE, through the use of the new class of estimators $\bar{H}^{(q)}(k)$, in (10) (the value $q=0.1$, in Figure 2, and the value $q=0$, in Figure 3). 

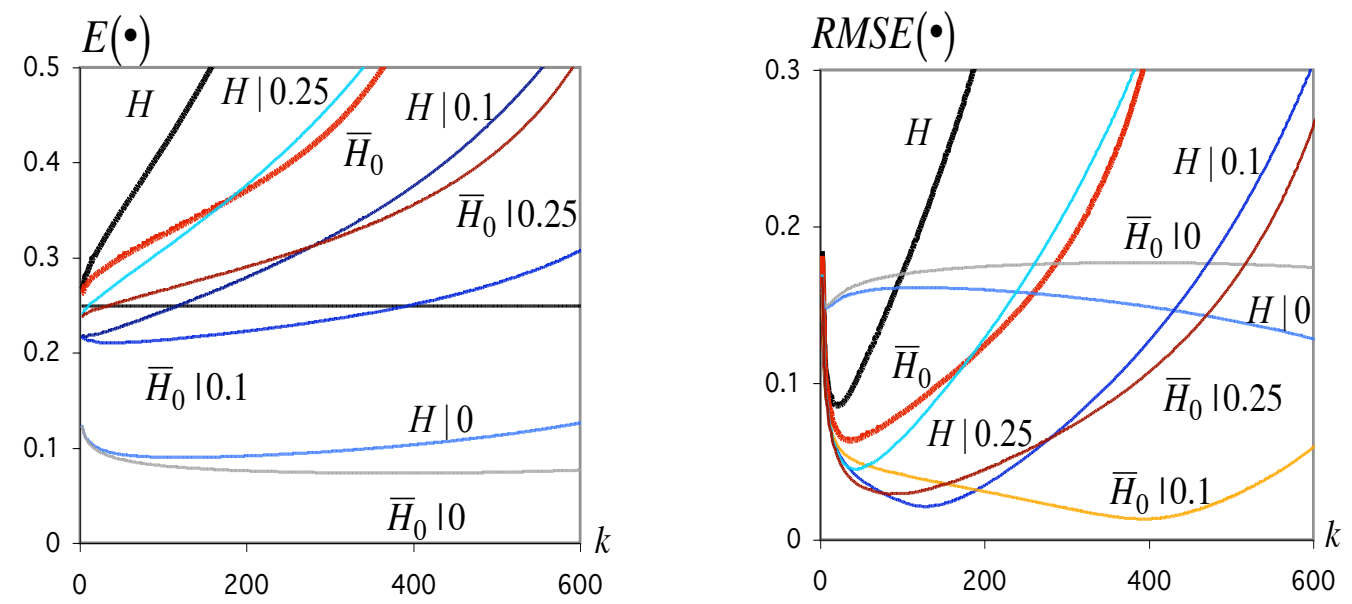

Figure 2: Patterns of mean values (left) and root mean squared errors (right), as functions of $k$, for an underlying Student $t_{4}$ parent $(\gamma=0.25, \rho=-0.5)$.
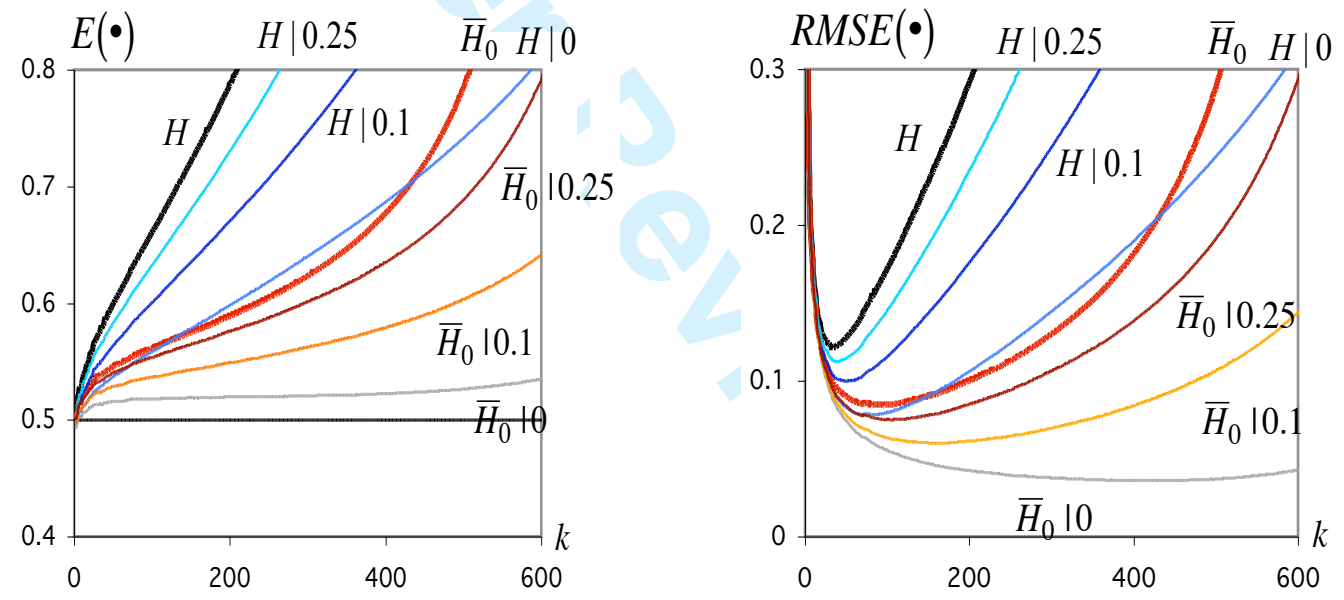

Figure 3: Patterns of mean values (left) and root mean squared errors (right), as functions of $k$, for an underlying Extreme Value parent with $\gamma=0.5(\rho=-0.5)$.

\subsection{Relative efficiencies and mean values at optimal levels}

Given a sample $\underline{\mathbf{X}}_{n}=\left(X_{1}, \ldots, X_{n}\right)$, let us denote $S(k)=S\left(k ; \underline{\mathbf{X}}_{n}\right)$ any statistic or r.v. dependent on $k$, the number of top o.s.'s to be used in an inferential procedure related with a parameter of extreme events. Just as mentioned before for the Hill estimator $H(k)$, in $(2)$, the OSF for $S(k)$ is denoted $k_{0}^{S} / n$, with $k_{0}^{S}:=\arg \min _{k} M S E(S(k))$. We shall now present, 
for $n=200,500,1000,2000$ and 5000, and with $\bullet$ denoting $H$ or $H \mid q$ or $\bar{H}_{\tau}$ or $\bar{H}_{\tau} \mid q$, $\tau=0,1$, the simulated $\operatorname{OSF}\left(k_{0}^{\bullet} / n\right)$, mean values $\left(E_{0}^{\bullet}\right)$ and relative efficiencies $\left(R E F F_{0}^{\bullet}\right)$ of the EVI-estimators under study, at their optimal levels. The search of the minimum MSE has been performed over the region of $k$-values between 1 and $[0.95 \times n]$. The MSE of $H$ is also provided so that it is possible to recover the MSE of any other EVI-estimator, due to the fact that for any EVI-estimator different from $H$, generally denoted $S$, the $R E F F_{0}^{S}$ indicator is given by

$$
R E F F_{0}^{S}:=\sqrt{\frac{M S E\left\{H\left(k_{0}^{H}\right)\right\}}{M S E\left\{S\left(k_{0}^{S}\right)\right\}}}=: \frac{R M S E_{0}^{H}}{R M S E_{0}^{S}} .
$$

Among the estimators considered, and for all $n$, the one providing the smallest squared bias and smallest MSE, or equivalently, the highest REFF is underlined and in bold. Tables 1, 2 and 3 are related with the underlying parents in I., II, and III., respectively, and 95\% confidence intervals are associated with all the estimates provided in the tables.

For an easier visualization, we present, in Figure 4, the REFF-indicators of the new PORTMVRB EVI-estimators, in (10), as well as of the PORT-Hill EVI-estimators in (5) and the MVRB EVI-estimators in (8), all at optimal levels, comparatively with the classical EVIestimators, in (2), also at their optimal level, for the underlying parents in I., II. and III.
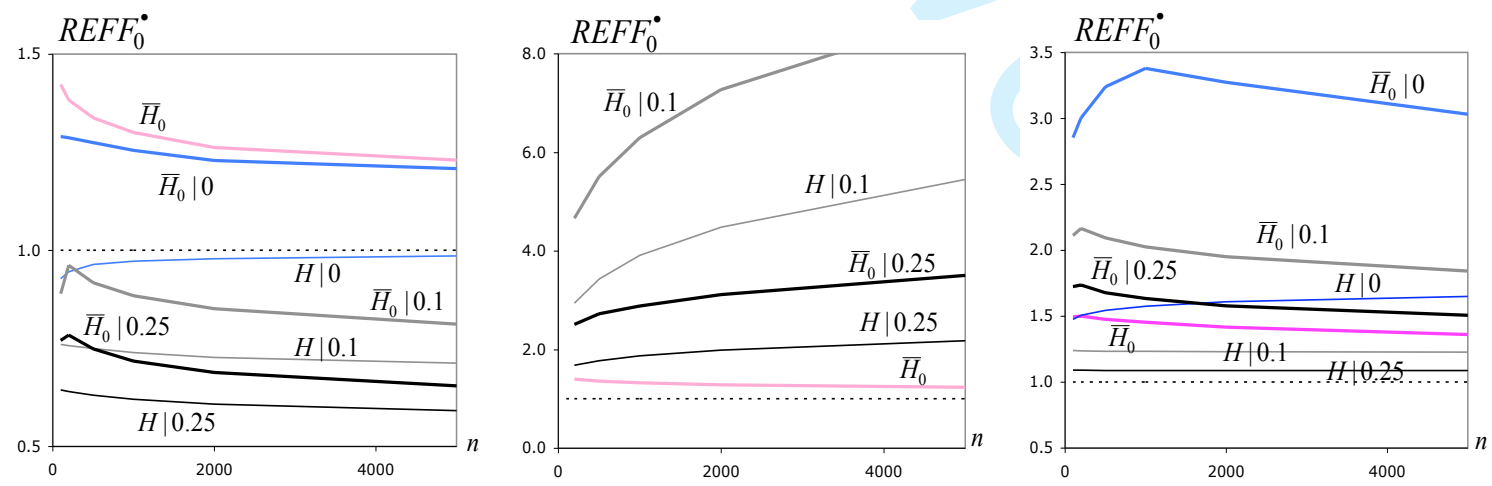

Figure 4: REFF-indicators for a Burr model with $\gamma=0.25$ and $\rho=-0.5$ (left), a Student $t_{4}$ model (center) and an EV model, with $\gamma=.5($ right $)$. 
Table 1: Simulated OSFs $\left(k_{0}^{\bullet} / n\right)$, mean values $\left(E_{0}^{\bullet}\right)$, MSE of $H_{0}$ and relative efficiency measures $\left(R E F F_{0}^{\bullet}\right)$ at optimal levels, for a Burr parent with $(\gamma, \rho)=(0.25,-0.5)$.

\begin{tabular}{|c|c|c|c|c|c|}
\hline$n$ & 200 & 500 & 1000 & 2000 & 5000 \\
\hline \multicolumn{6}{|c|}{$k_{0}^{\bullet} / n$} \\
\hline$H$ & $0.0778 \pm 0.0023$ & $0.0520 \pm 0.0017$ & $0.0388 \pm 0.0008$ & $0.0291 \pm 0.0009$ & $0.0187 \pm 0.0006$ \\
\hline$H \mid 0$ & $0.0720 \pm 0.0025$ & $0.0493 \pm 0.0016$ & $0.0376 \pm 0.0009$ & $0.0281 \pm 0.0010$ & $0.0181 \pm 0.0005$ \\
\hline$H \mid 0.1$ & $0.0540 \pm 0.0026$ & $0.0357 \pm 0.0011$ & $0.0260 \pm 0.0009$ & $0.0183 \pm 0.0006$ & $0.0115 \pm 0.0003$ \\
\hline$H \mid 0.25$ & $0.0425 \pm 0.0014$ & $0.0281 \pm 0.0010$ & $0.0202 \pm 0.0007$ & $0.0138 \pm 0.0005$ & $0.0084 \pm 0.0003$ \\
\hline $\bar{H}_{0}$ & $0.1573 \pm 0.0062$ & $0.1019 \pm 0.0031$ & $0.0719 \pm 0.0021$ & $0.0504 \pm 0.0019$ & $0.0299 \pm 0.0009$ \\
\hline $\bar{H}_{0} \mid 0$ & $0.1460 \pm 0.0059$ & $0.0961 \pm 0.0026$ & $0.0684 \pm 0.0024$ & $0.0479 \pm 0.0015$ & $0.0292 \pm 0.0010$ \\
\hline $\bar{H}_{0} \mid 0.1$ & $0.0938 \pm 0.0000$ & $0.0573 \pm 0.0020$ & $0.0396 \pm 0.0010$ & $0.0276 \pm 0.0012$ & $0.0156 \pm 0.0006$ \\
\hline $\bar{H}_{0} \mid 0.25$ & $0.0688 \pm 0.0000$ & $0.0426 \pm 0.0018$ & $0.0286 \pm 0.0007$ & $0.0196 \pm 0.0008$ & $0.0114 \pm 0.0004$ \\
\hline $\bar{H}_{1}$ & $0.0783 \pm 0.0023$ & $0.0531 \pm 0.0015$ & $0.0389 \pm 0.0008$ & $0.0291 \pm 0.0009$ & $0.0187 \pm 0.0006$ \\
\hline $\bar{H}_{1} \mid 0$ & $0.0728 \pm 0.0786$ & $0.0500 \pm 0.0345$ & $0.0378 \pm 0.0210$ & $0.0281 \pm 0.0139$ & $0.0183 \pm 0.0078$ \\
\hline $\bar{H}_{1} \mid 0.1$ & $0.0550 \pm 0.0000$ & $0.0358 \pm 0.0010$ & $0.0262 \pm 0.0009$ & $0.0183 \pm 0.0006$ & $0.0116 \pm 0.0003$ \\
\hline $\bar{H}_{1} \mid 0.25$ & $0.0425 \pm 0.0000$ & $0.0281 \pm 0.0010$ & $0.0202 \pm 0.0007$ & $0.0138 \pm 0.0005$ & $0.0114 \pm 0.0004$ \\
\hline \multicolumn{6}{|c|}{$E_{0}^{\bullet}$} \\
\hline$H$ & $0.3106 \pm 0.0012$ & $0.2965 \pm 0.0010$ & $0.2889 \pm 0.0006$ & $0.2827 \pm 0.0007$ & $0.2754 \pm 0.0006$ \\
\hline$H \mid 0$ & $0.3145 \pm 0.0011$ & $0.2986 \pm 0.0011$ & $0.2904 \pm 0.0007$ & $0.2835 \pm 0.0007$ & $0.2758 \pm 0.0005$ \\
\hline$H \mid 0.1$ & $0.3334 \pm 0.0021$ & $0.3158 \pm 0.0014$ & $0.3059 \pm 0.0010$ & $0.2972 \pm 0.0007$ & $0.2879 \pm 0.0006$ \\
\hline$H \mid 0.25$ & $0.3498 \pm 0.0016$ & $0.3296 \pm 0.0016$ & $0.3178 \pm 0.0011$ & $0.3070 \pm 0.0009$ & $0.2961 \pm 0.0008$ \\
\hline $\bar{H}_{0}$ & $\underline{\mathbf{0 . 2 9 7 4}} \pm 0.0010$ & $\underline{\mathbf{0 . 2 8 8 3}} \pm 0.0007$ & $\underline{\mathbf{0 . 2 8 2 5}} \pm 0.0004$ & $\underline{\mathbf{0 . 2 7 7 6}} \pm 0.0005$ & $\underline{\mathbf{0 . 2 7 1 8}} \pm 0.0004$ \\
\hline$\overline{\bar{H}}_{0} \mid 0$ & $0.3024 \pm 0.0011$ & $0.2907 \pm 0.0006$ & $0.2839 \pm 0.0005$ & $0.2784 \pm 0.0004$ & $0.2723 \pm 0.0004$ \\
\hline $\bar{H}_{0} \mid 0.1$ & $0.3220 \pm 0.0000$ & $0.3076 \pm 0.0009$ & $0.2994 \pm 0.0007$ & $0.2927 \pm 0.0008$ & $0.2844 \pm 0.0005$ \\
\hline $\bar{H}_{0} \mid 0.25$ & $0.3383 \pm 0.0000$ & $0.3215 \pm 0.0014$ & $0.3113 \pm 0.0007$ & $0.3032 \pm 0.0007$ & $0.2937 \pm 0.0006$ \\
\hline $\bar{H}_{1}$ & $0.3102 \pm 0.0011$ & $0.2969 \pm 0.0008$ & $0.2888 \pm 0.0006$ & $0.2827 \pm 0.0007$ & $0.2754 \pm 0.0006$ \\
\hline$\overline{\bar{H}}_{1} \mid 0$ & $0.3145 \pm 0.0011$ & $0.2988 \pm 0.0010$ & $0.2904 \pm 0.0007$ & $0.2835 \pm 0.0007$ & $0.2759 \pm 0.0005$ \\
\hline $\bar{H}_{1} \mid 0.1$ & $0.3341 \pm 0.0000$ & $0.3158 \pm 0.0013$ & $0.3060 \pm 0.0010$ & $0.2972 \pm 0.0007$ & $0.2881 \pm 0.0006$ \\
\hline $\bar{H}_{1} \mid 0.25$ & $0.3497 \pm 0.0000$ & $0.3295 \pm 0.0015$ & $0.3177 \pm 0.0011$ & $0.3069 \pm 0.0009$ & $0.2937 \pm 0.0006$ \\
\hline$M S E\left(H_{0}\right)$ & $0.0091 \pm 0.0001$ & $0.0052 \pm 0.0001$ & $0.0035 \pm 0.0000$ & $0.0023 \pm 0.0000$ & $0.0014 \pm 0.0000$ \\
\hline \multicolumn{6}{|c|}{$R E F F_{0}^{\bullet}$} \\
\hline$H \mid 0$ & $0.9454 \pm 0.0011$ & $0.9633 \pm 0.0010$ & $0.9718 \pm 0.0004$ & $0.9783 \pm 0.0005$ & $0.9852 \pm 0.0002$ \\
\hline$H \mid 0.1$ & $0.7568 \pm 0.0033$ & $0.7492 \pm 0.0029$ & $0.7387 \pm 0.0026$ & $0.7267 \pm 0.0017$ & $0.7122 \pm 0.0021$ \\
\hline$H \mid 0.25$ & $0.6396 \pm 0.0032$ & $0.6308 \pm 0.0032$ & $0.6198 \pm 0.0018$ & $0.6074 \pm 0.0018$ & $0.5909 \pm 0.0019$ \\
\hline $\bar{H}_{0}$ & $\underline{\mathbf{1 . 3 8 2 6}} \pm 0.0065$ & $\underline{\mathbf{1 . 3 3 7 3}} \pm 0.0052$ & $\underline{\mathbf{1 . 3 0 0 0}} \pm 0.0045$ & $\underline{\mathbf{1 . 2 6 2 3}} \pm 0.0045$ & $\underline{\mathbf{1 . 2 3 0 3}} \pm 0.0048$ \\
\hline $\bar{H}_{0} \mid 0$ & $1.2869 \pm 0.0060$ & $1.2744 \pm 0.0051$ & $1.2542 \pm 0.0053$ & $1.2288 \pm 0.0045$ & $1.2080 \pm 0.0047$ \\
\hline$\overline{\bar{H}}_{0} \mid 0.1$ & $0.9616 \pm 0.0000$ & $0.9173 \pm 0.0011$ & $0.8838 \pm 0.0006$ & $0.8509 \pm 0.0006$ & $0.8113 \pm 0.0014$ \\
\hline $\bar{H}_{0} \mid 0.25$ & $0.7840 \pm 0.0000$ & $0.7486 \pm 0.0021$ & $0.7176 \pm 0.0020$ & $0.6884 \pm 0.0014$ & $0.6546 \pm 0.0019$ \\
\hline $\bar{H}_{1}$ & $1.0049 \pm 0.0004$ & $1.0025 \pm 0.0002$ & $1.0015 \pm 0.0003$ & $1.0010 \pm 0.0000$ & $1.0005 \pm 0.0000$ \\
\hline $\bar{H}_{1} \mid 0$ & $0.9490 \pm 0.0009$ & $0.9652 \pm 0.0008$ & $0.9730 \pm 0.0003$ & $0.9791 \pm 0.0004$ & $0.9856 \pm 0.0003$ \\
\hline$\overline{\bar{H}}_{1} \mid 0.1$ & $0.7579 \pm 0.0000$ & $0.7500 \pm 0.0029$ & $0.7392 \pm 0.0026$ & $0.7269 \pm 0.0017$ & $0.7123 \pm 0.0021$ \\
\hline $\bar{H}_{1} \mid 0.25$ & $0.6401 \pm 0.0000$ & $0.6314 \pm 0.0032$ & $0.6202 \pm 0.0018$ & $0.6076 \pm 0.0018$ & $0.6546 \pm 0.0019$ \\
\hline
\end{tabular}


Table 2: Simulated OSFs $\left(k_{0}^{\bullet} / n\right)$, mean values $\left(E_{0}^{\bullet}\right)$, MSE of $H_{0}$ and relative efficiency measures $\left(R E F F_{0}^{\bullet}\right)$ at optimal levels, together with corresponding $95 \%$ confidence intervals, for a Student $t_{4}$ parent $(\gamma=0.25, \rho=-0.5)$.

\begin{tabular}{|c|c|c|c|c|c|}
\hline$n$ & 200 & 500 & 1000 & 2000 & 5000 \\
\hline \multicolumn{6}{|c|}{$k_{0}^{\bullet} / n$} \\
\hline$H$ & $0.0385 \pm 0.0017$ & $0.0258 \pm 0.0010$ & $0.0196 \pm 0.0007$ & $0.0144 \pm 0.0005$ & $0.0096 \pm 0.0003$ \\
\hline$H \mid 0.1$ & $0.1478 \pm 0.0027$ & $0.1345 \pm 0.0014$ & $0.1275 \pm 0.0012$ & $0.1247 \pm 0.0007$ & $0.1218 \pm 0.0005$ \\
\hline$H \mid 0.25$ & $0.0700 \pm 0.0019$ & $0.0534 \pm 0.0016$ & $0.0428 \pm 0.0012$ & $0.0348 \pm 0.0009$ & $0.0266 \pm 0.0007$ \\
\hline $\bar{H}_{0}$ & $0.0863 \pm 0.0033$ & $0.0503 \pm 0.0028$ & $0.0352 \pm 0.0011$ & $0.0249 \pm 0.0007$ & $0.0151 \pm 0.0004$ \\
\hline $\bar{H}_{0} \mid 0.1$ & $0.3718 \pm 0.0030$ & $0.3882 \pm 0.0020$ & $0.3934 \pm 0.0013$ & $0.3970 \pm 0.0008$ & $0.3988 \pm 0.0004$ \\
\hline $\bar{H}_{0} \mid 0.25$ & $0.1375 \pm 0.0045$ & $0.1067 \pm 0.0022$ & $0.0899 \pm 0.0023$ & $0.0742 \pm 0.0015$ & $0.0598 \pm 0.0009$ \\
\hline $\bar{H}_{1}$ & $0.0435 \pm 0.0019$ & $0.0283 \pm 0.0012$ & $0.0200 \pm 0.0007$ & $0.0144 \pm 0.0005$ & $0.0096 \pm 0.0003$ \\
\hline $\bar{H}_{1} \mid 0.1$ & $0.1533 \pm 0.0026$ & $0.1395 \pm 0.0018$ & $0.1314 \pm 0.0016$ & $0.1287 \pm 0.0009$ & $0.1255 \pm 0.0005$ \\
\hline $\bar{H}_{1} \mid 0.25$ & $0.0703 \pm 0.0018$ & $0.0540 \pm 0.0016$ & $0.0430 \pm 0.0013$ & $0.0352 \pm 0.0010$ & $0.0267 \pm 0.0007$ \\
\hline \multicolumn{6}{|c|}{$E_{0}^{\bullet}$} \\
\hline$H$ & $0.3392 \pm 0.0026$ & $0.3167 \pm 0.0016$ & $0.3055 \pm 0.0013$ & $0.2959 \pm 0.0009$ & $0.2862 \pm 0.0007$ \\
\hline$H \mid 0.1$ & $0.2634 \pm 0.0009$ & $0.2564 \pm 0.0004$ & $0.2533 \pm 0.0004$ & $0.2520 \pm 0.0002$ & $0.2508 \pm 0.0002$ \\
\hline$H \mid 0.25$ & $0.2935 \pm 0.0012$ & $0.2806 \pm 0.0011$ & $0.2728 \pm 0.0008$ & $0.2672 \pm 0.0006$ & $0.2613 \pm 0.0005$ \\
\hline $\bar{H}_{0}$ & $0.3104 \pm 0.0009$ & $0.3005 \pm 0.0013$ & $0.2939 \pm 0.0008$ & $0.2879 \pm 0.0006$ & $0.2805 \pm 0.0004$ \\
\hline $\bar{H}_{0} \mid 0.1$ & $\underline{\mathbf{0 . 2 4 9 8}} \pm 0.0005$ & $\underline{\mathbf{0 . 2 4 9 9}} \pm 0.0004$ & $\underline{\mathbf{0 . 2 4 9 8}} \pm 0.0002$ & $\underline{\mathbf{0 . 2 5 0 0}} \pm 0.0001$ & $\underline{\mathbf{0 . 2 5 0 0}} \pm 0.0000$ \\
\hline $\bar{H}_{0} \mid 0.25$ & $0.2783 \pm 0.0012$ & $0.2686 \pm 0.0006$ & $0.2641 \pm 0.0006$ & $0.2599 \pm 0.0003$ & $0.2561 \pm 0.0002$ \\
\hline $\bar{H}_{1}$ & $0.3365 \pm 0.0024$ & $0.3181 \pm 0.0015$ & $0.3052 \pm 0.0012$ & $0.2956 \pm 0.0009$ & $0.2860 \pm 0.0007$ \\
\hline $\bar{H}_{1} \mid 0.1$ & $0.2634 \pm 0.0008$ & $0.2565 \pm 0.0006$ & $0.2532 \pm 0.0005$ & $0.2520 \pm 0.0003$ & $0.2508 \pm 0.0002$ \\
\hline $\bar{H}_{1} \mid 0.25$ & $0.2930 \pm 0.0012$ & $0.2806 \pm 0.0010$ & $0.2728 \pm 0.0008$ & $0.2674 \pm 0.0006$ & $0.2612 \pm 0.0005$ \\
\hline$M S E_{0}^{H}$ & $0.0205 \pm 0.0003$ & $0.0112 \pm 0.0001$ & $0.0073 \pm 0.0001$ & $0.0048 \pm 0.0000$ & $0.0029 \pm 0.0000$ \\
\hline \multicolumn{6}{|c|}{$R E F F_{0}^{\bullet}$} \\
\hline$H \mid 0.1$ & $2.9437 \pm 0.0206$ & $3.4305 \pm 0.0218$ & $3.9106 \pm 0.0209$ & $4.4822 \pm 0.0305$ & $5.4522 \pm 0.0335$ \\
\hline$H \mid 0.25$ & $1.6823 \pm 0.0083$ & $1.7745 \pm 0.0072$ & $1.8702 \pm 0.0077$ & $1.9850 \pm 0.0081$ & $2.1777 \pm 0.0092$ \\
\hline $\bar{H}_{0}$ & $1.3982 \pm 0.0084$ & $1.3615 \pm 0.0053$ & $1.3223 \pm 0.0057$ & $1.2834 \pm 0.0057$ & $1.2358 \pm 0.0048$ \\
\hline $\bar{H}_{0} \mid 0.1$ & $\underline{\mathbf{4 . 6 5 9 8}} \pm 0.0358$ & $\underline{\mathbf{5 . 5 0 3 6}} \pm 0.0348$ & $\underline{\mathbf{6 . 2 9 5 9}} \pm 0.0364$ & $\underline{\mathbf{7 . 2 6 9 2}} \pm 0.0563$ & $\underline{\mathbf{8 . 8 4 7 8}} \pm 0.0457$ \\
\hline $\bar{H}_{0} \mid 0.25$ & $2.5115 \pm 0.0167$ & $2.7219 \pm 0.0153$ & $2.8846 \pm 0.0169$ & $3.1153 \pm 0.0215$ & $3.5054 \pm 0.0174$ \\
\hline $\bar{H}_{1}$ & $1.0443 \pm 0.0033$ & $1.0163 \pm 0.0013$ & $1.0078 \pm 0.0006$ & $1.0039 \pm 0.0002$ & $1.0016 \pm 0.0002$ \\
\hline $\bar{H}_{1} \mid 0.1$ & $2.9950 \pm 0.0216$ & $3.4867 \pm 0.0220$ & $3.9712 \pm 0.0205$ & $4.5530 \pm 0.0325$ & $5.5340 \pm 0.0349$ \\
\hline $\bar{H}_{1} \mid 0.25$ & $1.6917 \pm 0.0083$ & $1.7820 \pm 0.0073$ & $1.8758 \pm 0.0079$ & $1.9898 \pm 0.0081$ & $2.1816 \pm 0.0092$ \\
\hline
\end{tabular}

Figure 5 is equivalent to Figure 4, but with the simulated mean values of the EVIestimators at optimal levels.

Regarding the REFF-indicators, we would like to draw the following comments:

- For models like the Burr, with a left endpoint equal to zero, we cannot acchieve any improvement with the shifted estimators. 
Table 3: Simulated OSFs $\left(k_{0}^{\bullet} / n\right)$, mean values $\left(E_{0}^{\bullet}\right)$, MSE of $H_{0}$ and relative efficiency measures $\left(R E F F_{0}^{\bullet}\right)$ at optimal levels, together with corresponding $95 \%$ confidence intervals, for a $E V_{0.5}$ parent $(\gamma=-\rho=0.5)$.

\begin{tabular}{|c|c|c|c|c|c|}
\hline$n$ & 200 & 500 & 1000 & 2000 & 5000 \\
\hline \multicolumn{6}{|c|}{$k_{0}^{\bullet} / n$} \\
\hline$H$ & $0.0663 \pm 0.0021$ & $0.0465 \pm 0.0008$ & $0.0351 \pm 0.0010$ & $0.0254 \pm 0.0006$ & $0.0173 \pm 0.0006$ \\
\hline$H \mid 0$ & $0.1338 \pm 0.0039$ & $0.1003 \pm 0.0030$ & $0.0767 \pm 0.0022$ & $0.0616 \pm 0.0022$ & $0.0427 \pm 0.0012$ \\
\hline$H \mid 0.1$ & $0.0955 \pm 0.0031$ & $0.0666 \pm 0.0020$ & $0.0503 \pm 0.0011$ & $0.0370 \pm 0.0009$ & $0.0256 \pm 0.0006$ \\
\hline$H \mid 0.25$ & $0.0790 \pm 0.0030$ & $0.0542 \pm 0.0020$ & $0.0400 \pm 0.0013$ & $0.0306 \pm 0.0006$ & $0.0204 \pm 0.0005$ \\
\hline $\bar{H}_{0}$ & $0.2045 \pm 0.0065$ & $0.1223 \pm 0.0044$ & $0.0886 \pm 0.0029$ & $0.0605 \pm 0.0025$ & $0.0372 \pm 0.0011$ \\
\hline$\overline{\bar{H}}_{0} \mid 0$ & $0.4818 \pm 0.0082$ & $0.4504 \pm 0.0056$ & $0.4193 \pm 0.0080$ & $0.3764 \pm 0.0079$ & $0.3350 \pm 0.0067$ \\
\hline $\bar{H}_{0} \mid 0.1$ & $0.2735 \pm 0.0066$ & $0.2019 \pm 0.0048$ & $0.1505 \pm 0.0049$ & $0.1146 \pm 0.0036$ & $0.0700 \pm 0.0028$ \\
\hline $\bar{H}_{0} \mid 0.25$ & $0.1919 \pm 0.0076$ & $0.1348 \pm 0.0042$ & $0.1014 \pm 0.0030$ & $0.0718 \pm 0.0028$ & $0.0446 \pm 0.0016$ \\
\hline$\overline{\bar{H}}_{1}$ & $0.0803 \pm 0.0028$ & $0.0513 \pm 0.0021$ & $0.0367 \pm 0.0015$ & $0.0259 \pm 0.0007$ & $0.0174 \pm 0.0006$ \\
\hline $\bar{H}_{1} \mid 0$ & $0.1440 \pm 0.0423$ & $0.1053 \pm 0.0326$ & $0.0805 \pm 0.0302$ & $0.0630 \pm 0.0247$ & $0.0436 \pm 0.0157$ \\
\hline $\bar{H}_{1} \mid 0.1$ & $0.0988 \pm 0.0031$ & $0.0679 \pm 0.0021$ & $0.0510 \pm 0.0010$ & $0.0376 \pm 0.0010$ & $0.0257 \pm 0.0006$ \\
\hline $\bar{H}_{1} \mid 0.25$ & $0.0806 \pm 0.0049$ & $0.0550 \pm 0.0020$ & $0.0400 \pm 0.0013$ & $0.0309 \pm 0.0006$ & $0.0446 \pm 0.0016$ \\
\hline \multicolumn{6}{|c|}{$E_{0}^{\bullet}$} \\
\hline$H$ & $0.6236 \pm 0.0033$ & $0.5957 \pm 0.0011$ & $0.5791 \pm 0.0016$ & $0.5647 \pm 0.0010$ & $0.5513 \pm 0.0010$ \\
\hline$H \mid 0$ & $0.5814 \pm 0.0017$ & $0.5611 \pm 0.0014$ & $0.5485 \pm 0.0010$ & $0.5401 \pm 0.0009$ & $0.5303 \pm 0.0006$ \\
\hline$H \mid 0.1$ & $0.6001 \pm 0.0023$ & $0.5769 \pm 0.0015$ & $0.5638 \pm 0.0009$ & $0.5524 \pm 0.0009$ & $0.5419 \pm 0.0006$ \\
\hline$H \mid 0.25$ & $0.6155 \pm 0.0035$ & $0.5879 \pm 0.0021$ & $0.5717 \pm 0.0015$ & $0.5607 \pm 0.0009$ & $0.5477 \pm 0.0007$ \\
\hline $\bar{H}_{0}$ & $0.5733 \pm 0.0016$ & $0.5643 \pm 0.0014$ & $0.5579 \pm 0.0010$ & $0.5499 \pm 0.0009$ & $0.5412 \pm 0.0006$ \\
\hline $\bar{H}_{0} \mid 0$ & $\underline{\mathbf{0 . 5 3 4 6}} \pm 0.0007$ & $\underline{\mathbf{0 . 5 2 7 0}} \pm 0.0003$ & $\underline{\mathbf{0 . 5 2 3 1}} \pm 0.0003$ & $\underline{\mathbf{0 . 5 2 2 2}} \pm 0.0002$ & $\underline{\mathbf{0 . 5 2 1 1}} \pm 0.0001$ \\
\hline $\bar{H}_{0} \mid 0.1$ & $0.5596 \pm 0.0011$ & $0.5495 \pm 0.0007$ & $0.5427 \pm 0.0006$ & $0.5382 \pm 0.0006$ & $0.5315 \pm 0.0005$ \\
\hline $\bar{H}_{0} \mid 0.25$ & $0.5769 \pm 0.0019$ & $0.5619 \pm 0.0010$ & $0.5536 \pm 0.0009$ & $0.5459 \pm 0.0008$ & $0.5374 \pm 0.0006$ \\
\hline $\bar{H}_{1}$ & $0.6188 \pm 0.0032$ & $0.5954 \pm 0.0021$ & $0.5788 \pm 0.0020$ & $0.5645 \pm 0.0011$ & $0.5512 \pm 0.0010$ \\
\hline $\bar{H}_{1} \mid 0$ & $0.5827 \pm 0.0019$ & $0.5618 \pm 0.0013$ & $0.5494 \pm 0.0008$ & $0.5403 \pm 0.0009$ & $0.5304 \pm 0.0005$ \\
\hline $\bar{H}_{1} \mid 0.1$ & $0.6008 \pm 0.0021$ & $0.5770 \pm 0.0015$ & $0.5638 \pm 0.0008$ & $0.5527 \pm 0.0009$ & $0.5419 \pm 0.0006$ \\
\hline $\bar{H}_{1} \mid 0.25$ & $0.6157 \pm 0.0060$ & $0.5878 \pm 0.0021$ & $0.5714 \pm 0.0015$ & $0.5609 \pm 0.0009$ & $0.5374 \pm 0.0006$ \\
\hline$M S E\left(H_{0}\right)$ & $0.0409 \pm 0.0005$ & $0.0227 \pm 0.0002$ & $0.0149 \pm 0.0001$ & $0.0099 \pm 0.0001$ & $0.0059 \pm 0.0000$ \\
\hline \multicolumn{6}{|c|}{$R E F F_{0}^{\bullet}$} \\
\hline$H \mid 0$ & $1.5056 \pm 0.0097$ & $1.5443 \pm 0.0058$ & $1.5752 \pm 0.0066$ & $1.6080 \pm 0.0066$ & $1.6494 \pm 0.0079$ \\
\hline$H \mid 0.1$ & $1.2348 \pm 0.0057$ & $1.2320 \pm 0.0038$ & $1.2316 \pm 0.0045$ & $1.2288 \pm 0.0036$ & $1.2275 \pm 0.0041$ \\
\hline$H \mid 0.25$ & $1.0912 \pm 0.0020$ & $1.0878 \pm 0.0021$ & $1.0872 \pm 0.0026$ & $1.0859 \pm 0.0018$ & $1.0860 \pm 0.0020$ \\
\hline $\bar{H}_{0}$ & $1.5009 \pm 0.0097$ & $1.4760 \pm 0.0059$ & $1.4519 \pm 0.0057$ & $1.4167 \pm 0.0057$ & $1.3590 \pm 0.0052$ \\
\hline $\bar{H}_{0} \mid 0$ & $\underline{\mathbf{3 . 0 0 3 8}} \pm 0.0192$ & $\underline{\mathbf{3 . 2 3 8 5}} \pm 0.0149$ & $\underline{\mathbf{3 . 3 7 9 2}} \pm 0.0143$ & $\underline{\mathbf{3 . 2 7 2 6}} \pm 0.0145$ & $\underline{\mathbf{3 . 0 3 0 8}} \pm 0.0114$ \\
\hline $\bar{H}_{0} \mid 0.1$ & $2.1648 \pm 0.0149$ & $2.0932 \pm 0.0120$ & $2.0248 \pm 0.0094$ & $1.9490 \pm 0.0079$ & $1.8406 \pm 0.0066$ \\
\hline $\bar{H}_{0} \mid 0.25$ & $1.7370 \pm 0.0198$ & $1.6770 \pm 0.0084$ & $1.6322 \pm 0.0075$ & $1.5778 \pm 0.0067$ & $1.5068 \pm 0.0065$ \\
\hline $\bar{H}_{1}$ & $1.0624 \pm 0.0047$ & $1.0245 \pm 0.0012$ & $1.0128 \pm 0.0009$ & $1.0066 \pm 0.0005$ & $1.0030 \pm 0.0003$ \\
\hline $\bar{H}_{1} \mid 0$ & $1.5283 \pm 0.0097$ & $1.5594 \pm 0.0057$ & $1.5851 \pm 0.0068$ & $1.6160 \pm 0.0067$ & $1.6544 \pm 0.0080$ \\
\hline $\bar{H}_{1} \mid 0.1$ & $1.2447 \pm 0.0060$ & $1.2377 \pm 0.0039$ & $1.2356 \pm 0.0046$ & $1.2313 \pm 0.0037$ & $1.2290 \pm 0.0041$ \\
\hline $\bar{H}_{1} \mid 0.25$ & $1.0980 \pm 0.0053$ & $1.0929 \pm 0.0023$ & $1.0901 \pm 0.0028$ & $1.0880 \pm 0.0018$ & $1.5068 \pm 0.0065$ \\
\hline
\end{tabular}



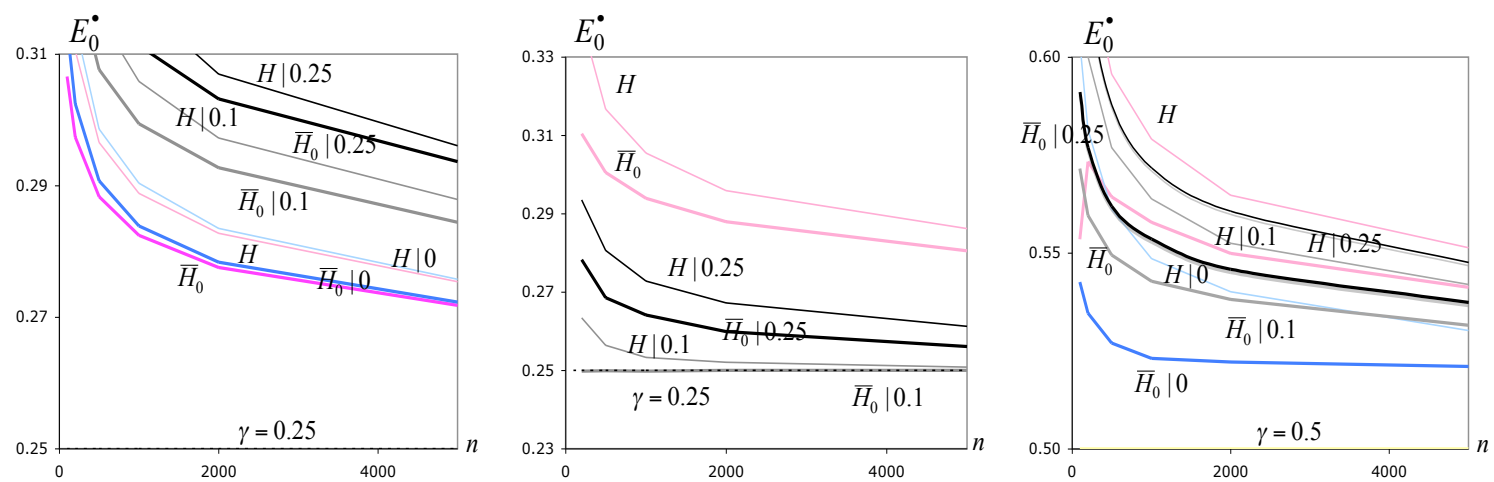

Figure 5: Mean values at optimal levels for a Burr model with $\gamma=0.25$ and $\rho=-0.5$ (left), a Student $t_{4}$ model (center and an EV model, with $\gamma=.5$ (right).

- For a model like the Student $t_{\nu}$, here illustrated for $\nu=4$, the PORT-MVRB EVIestimators have the best performance for all $n$, if $q=0.1$. However, even the PORTHill EVI-estimators associated with $q=0.1$ exhibit also a high efficiency.

- For an underlying EV model, we reach a clear improvement in the estimation of $\gamma$, whenever we consider the PORT estimators, based on the MVRB EVI-estimator. The REFF-indicators of the PORT-MVRB EVI-estimators associated with $q=0$ are the highest ones for all $n$. Note however that even the PORT-Hill estimators provide REFF-indicators higher than one for all $n$, with the highest indicator associated with $q=0$ (a shift induced by the minimum of the available sample).

It is also clear from Figures 4 and 5 that there is not a full agreement between REFF and BIAS indicators, but the discrepancies are moderate. Regarding bias at optimal levels, we can draw the following comments:

- For the simulated Burr model the MVRB EVI-estimators exhibit the smallest bias for all $n$, but not a long way from the PORT EVI-estimator associated with $q=0$, as expected.

- For the Student $t_{4}$ model, the PORT-MVRB EVI-estimator based on $q=0.1$ has a quite interesting bias pattern, practically equal to zero for all $n$.

- For an EV model with $\gamma=0.5$ and for all $n$, the smallest bias is achieved by the 
PORT-MVRB EVI-estimator based on the shifted version of $\bar{H}$, associated with $q=0$. This also happens for other values of $\gamma$.

- Note also the over-estimation achieved by all EVI-estimators considered, with an exception for the PORT-MVRB EVI-estimators associated with $q=0.1$ in a Student underlying parent.

In Figure 6, we still present the REFF-indicators of the EVI-estimators, in (5), (8) and (10), comparatively with the classical EVI-estimators, in (2), all at optimal levels, for an EV model with $\gamma=1(\rho=-1)$ and a Fréchet model with $\gamma=0.25(\rho=-1$, as well). The Fréchet d.f. is $\Phi_{1 / \gamma}(x)=\exp \left(-x^{-1 / \gamma}\right), x \geq 0$.
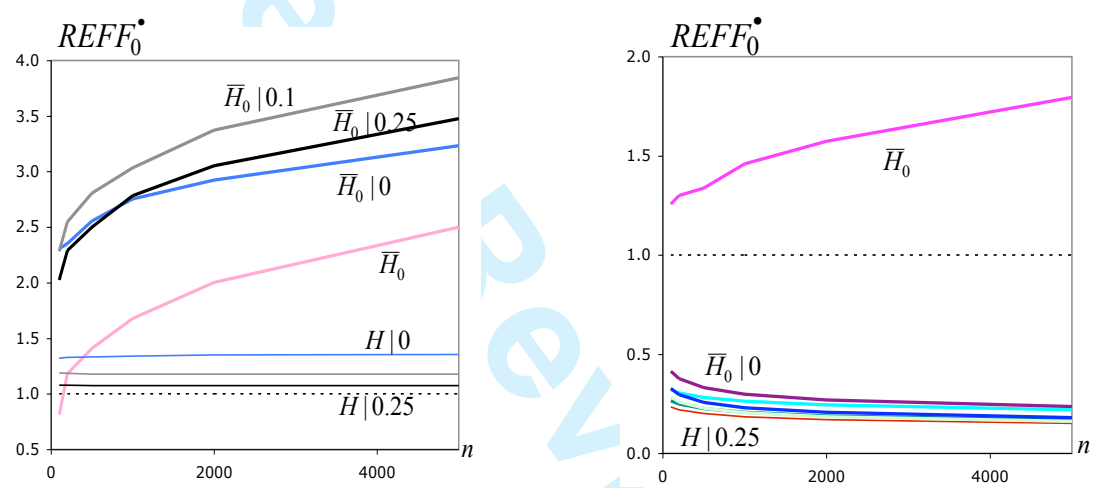

Figure 6: REFF-indicators for a GEV model with $\gamma=1$ (left) and a Fréchet model with $\gamma=0.25$ (right), both with $\rho=-1$.

Figure 7 is equivalent to Figure 6 , but with the mean values at optimal levels, for the same extra models as in Figure 6.

In summary we may draw the following final conclusions:

1. If the underlying model has a finite left endpoint greater than or equal to zero, the PORT estimators can never bit the original estimators.

2. For the range of $\rho$-values close to zero (greater than -1 , say), the use of $\tau=1$ provides results only slightly better than the ones associated with the classical estimator. If $|\rho| \geq 1$, the choice $\tau=1$ is more convenient, as already detected in other papers on the subject. 

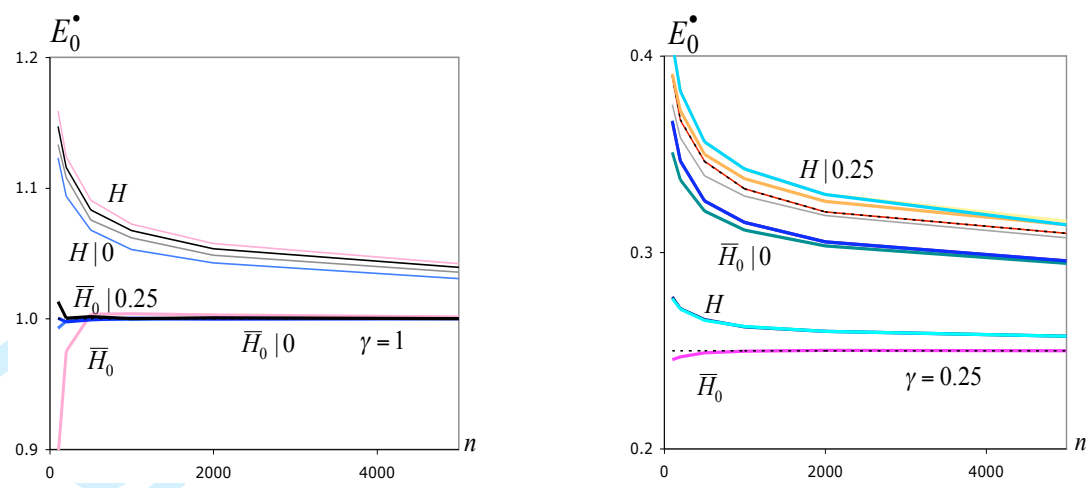

Figure 7: Mean values at optimal levels for a GEV model with $\gamma=1$ (left) and a Fréchet model with $\gamma=0.25$ (right), both with $\rho=-1$.

3. For parents with an infinite left endpoint, like the Student parents, or a left endpoint smaller than zero, like the EV parents, the best performance regarding efficiency is attained by the new estimators for an adequate value of $q$. Such a $q$ depends on the underlying model and on the sample size $n$. A similar comment applies to bias reduction.

\section{Data-driven choice of tuning parameters and case studies}

With the notation $X_{0: n}=0$, and with $\bar{H}$ and $\bar{H}^{(q)}$, given in (8) and (10), respectively, we can consider that $\bar{H}=\bar{H}^{(q)}$ for $q=-1 / n\left(n_{q}=0\right)$. Our interest lies then on the estimation of $\gamma$ through $\bar{H}^{(q)}$, in (10), now also including $\bar{H}$, in (8). Based on the stability on $k$ of the MVRB estimates, in (8), and the PORT-MVRB estimates, in (10), for adequate values of $q$, we propose now a method for an adaptive heuristic estimation of $\gamma$.

\subsection{An algorithm for the heuristic choice of $k$ and $q$} Algorithm.

1. Given an observed sample $\left(x_{1}, x_{2}, \ldots, x_{n}\right)$, consider, for $q=-1 / n, 0(0.1) 0.5$, the 
observed sample of excesses, $\underline{\mathbf{x}}_{n}^{(q)}$, with $\underline{\mathbf{X}}_{n}^{(q)}$ given in (4), and compute $\hat{\rho} \equiv \hat{\rho}_{0}=$ $\hat{\rho}_{0}\left(k_{1} ; \underline{\mathbf{x}}_{n}^{(q)}\right)$ and $\hat{\beta} \equiv \hat{\beta}_{0}:=\hat{\beta}_{\hat{\rho}_{0}}\left(k_{1} ; \underline{\mathbf{x}}_{n}^{(q)}\right), \hat{\rho}_{0}(k), k_{1}$ and $\hat{\beta}_{\hat{\rho}}(k)$ given in (14), (17) and (18), respectively.

2. Next compute, for $k=1,2, \ldots, n-[n q]-1$, the observed values of $\bar{H}_{0}^{(q)}(k)$, with $\bar{H}^{(q)}(k)$ given in (10) (note that, as mentioned before and with $\bar{H}$ given in (8), $\left.\bar{H}^{(-1 / n)} \equiv \bar{H}\right)$.

3. Obtain $j_{0}$, the minimum value of $j$, a non-negative integer, such that $a_{k}(j)=$ $\left[\bar{H}_{0}^{(q)}(k) \times 10^{j}\right], k=1,2, \cdots, n-[n q]-1$, has distinct elements (in the applications, in Section 4.2 , we were led to $j_{0}=1$ for all data sets considered).

4. For each $q$ and for $k>\hat{k}_{0}^{H}$, with $\hat{k}_{0}^{H}$ given in (13), consider as an estimate of $\gamma$ the equal consecutive values $\bar{H}_{0}^{(q)}(k), k \in \mathcal{K}_{q}$, with $\mathcal{K}_{q}=\left[k_{\text {min }}^{(q)}, k_{\text {max }}^{(q)}\right]$, to which is associated the largest size $l_{q}:=\# \mathcal{K}_{q}=k_{\text {max }}^{(q)}-k_{\text {min }}^{(q)}$.

5. Choose next $q_{0}:=\arg \max _{q} l_{q}$.

6. Consider all those estimates, $\bar{H}_{0}^{\left(q_{0}\right)}(k), k_{\text {min }}^{\left(q_{0}\right)} \leq k \leq k_{\text {max }}^{\left(q_{0}\right)}$, now with an extra decimal place, i.e., $\bar{H}^{\left(q_{0}\right)}(k)=a_{k}\left(j_{0}+1\right) / 10^{j_{0}+1}$. Count the frequencies associated to those estimates and obtain the mode $\eta_{0} \equiv \eta_{q_{0}}$ of these values. Let us denote $\mathcal{K}^{*}$ the set of $k$-values corresponding to those estimates.

7. In order to enlarge the size of the associated CI, take $k_{0}$ as the minimum of $\mathcal{K}^{*}$ and the adaptive EVI-estimate $\hat{\gamma}=\bar{H}_{0}^{\left(q_{0}\right)}\left(k_{0}\right)$.

\subsection{Case studies and a simulated sample}

\subsubsection{A data set in the field of insurance}

We shall first consider an illustration of the performance of the Algorithm, when applied to the analysis of automobile claim amounts exceeding 1,200,000 Euro over the period 19882001, gathered from several European insurance companies co-operating with the same re-insurer (Secura Belgian Re). This data set was already studied in Beirlant et al. (2004), Vandewalle and Beirlant (2006) and Beirlant et al. (2008) as an example to excess-of-loss 
reinsurance rating and heavy-tailed distributions in car insurance. As detected before, and can be seen from the histogram in Figure 8, the right-tail is quite heavy. The data is censored at the left, with a finite positive left endpoint, and we thus expect to have no improvement through the use of the PORT methodology, and we expect to be led by the Algorithm to the choice of the MVRB EVI-estimator, $\bar{H}$, in (8).

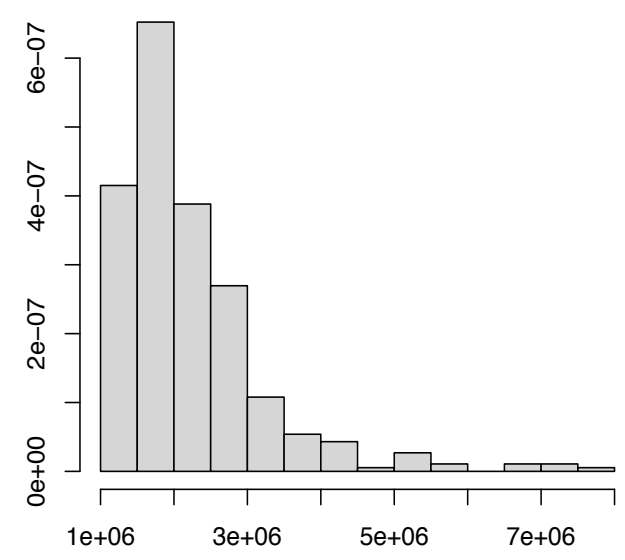

Figure 8: A histogram associated with the SECURA data.

In Figure 9, we present, for the original sample, the sample path of the $\rho$-estimates in (14) and (16), as function of $k$, together with the sample paths of the $\beta$-estimators in (18), also for $\tau=0$ and $\tau=1$. We have $n=n_{0}=371$, and we got $\widehat{\rho}=\widehat{\rho}_{0}(368)=-0.74$. We were next led to the estimate $\widehat{\beta}=0.80$.

In Figure 10, we present the estimates of $\gamma$, provided by $H$ and $\bar{H}^{(q)}, q=-1 / n, 0$ and 0.2 , with $H$ and $\bar{H}^{(q)}$ given in (2) and (10), respectively. Regarding the EVI-estimation, note that the Hill estimator $H(k)$, in (2), is unbiased for the estimation of the extreme value index $\gamma$ only when the underlying model is a strict Pareto model. Otherwise, i.e. when we have only Pareto-like tails, as happens here and can be seen from Figure 10, it exhibits a quite relevant bias. The MVRB EVI-estimators, $\bar{H}$, in (8), which are "asymptotically unbiased", have a smaller bias, exhibit more stable sample paths as functions of $k$, and enable us to take a decision upon the estimate of $\gamma$ and other parameters of extreme events to be 


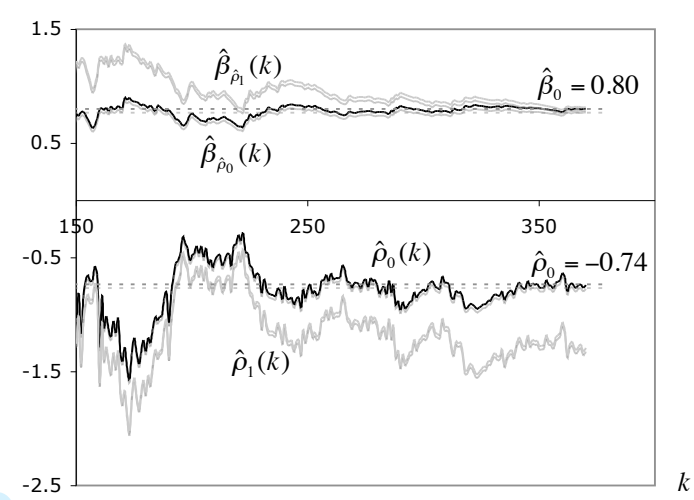

Figure 9: Estimates of the shape second-order parameter $\rho$ and of the scale second-order parameter $\beta$ for the SECURA data.

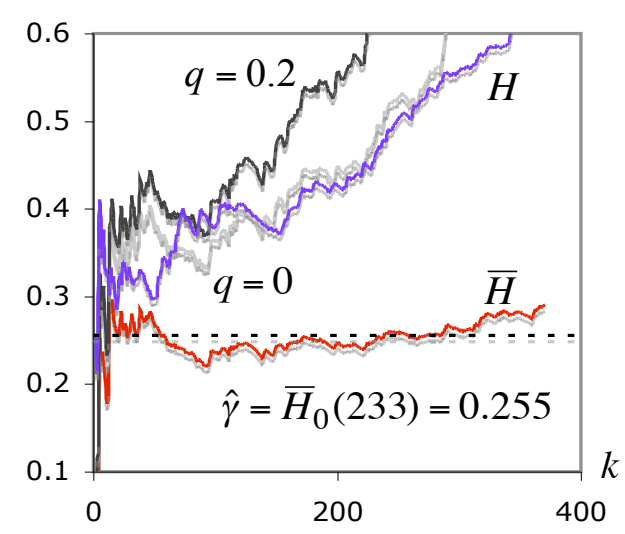

Figure 10: EVI-estimates, as a function of $k$, for the SECURA data, as well as the adaptive estimates obtained through the Algorithm.

used, with the help of any heuristic stability criterion, like the "largest run" suggested in Gomes et al. (2004), and the one written algorithmically in Section 4.1, regarding adaptive PORT-MVRB EVI-estimation.

- In Step 5. of the Algorithm, we have been led to the choice $q_{0}=-1 / n$, associated with a run of size 140 of a $\gamma$-estimate equal to 0.3. In Step 6., we got a mode $\eta_{0}=59$ for the $\gamma$-estimate $0.26, k_{0}=233$ and the adaptive PORT-MVRB estimate $\hat{\gamma}=0.255$. The associated 99\% MVRB-confidence interval for $\gamma$ is $(0.218,0.307)$, with a size equal to 0.089 . 
- The estimate of $k_{0}^{H}$, provided in $(13)$, is $\hat{k}_{0}^{H}=54$ and $H(54)=0.320$. The approximate $99 \%$ confidence interval is then $(0.219,0.420)$, with a size equal to $0.201>0.089$.

\subsubsection{A data set in the field of finance}

We have also considered the performance of the Algorithm, when applied to the analysis of the log-returns associated with one of the four sets of financial data considered in Gomes and Pestana (2007), the daily closing values of the Microsoft Corp. (MSFT). Although there is some increasing trend in the volatility, stationarity and weak dependence are assumed, under the same considerations as in Drees (2003). For this data set, we now present, in Figure 11, a box-and-whiskers' plot of the available data. From Figure 11, we immediately see that the underlying model has heavy left and right tails. This is the usual situation for log-returns data, and leads to the elimination of the estimators associated with $q=0$, due to their inconsistency. Figure 12 is similar to Figure 10, now for the MSFT data..

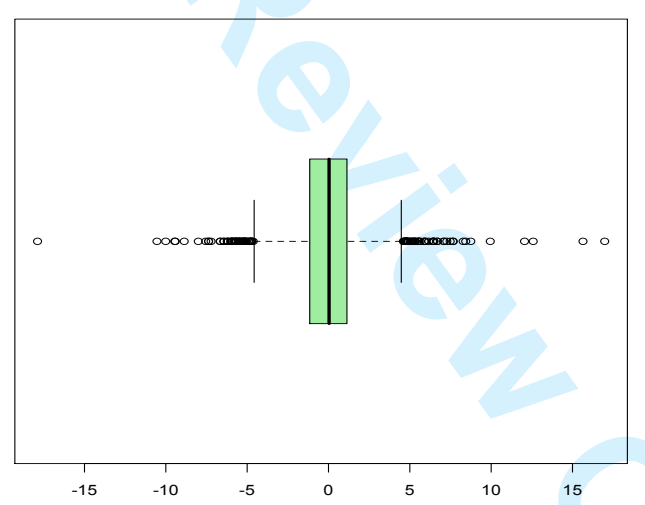

Figure 11: Box-and-whiskers plot associated with the MSFT data.

- The number of positive elements in the available sample of log-returns is now $n_{0}=$ 882. We were led to the $\rho$-estimate $\hat{\rho} \equiv \hat{\rho}_{0}=-0.72$, obtained at the level $k_{1}=$ $\left[n_{0}^{0.999}\right]=876$. The associated $\beta$-estimate is $\hat{\beta} \equiv \hat{\beta}_{0}=1.02$.

- In Step 5. of the Algorithm, we have been led to the choice $q_{0}=0.1$, associated with a run of size 810 of a $\gamma$-estimate equal to 0.2. In Step 6. of Algorithm I, we got a mode 


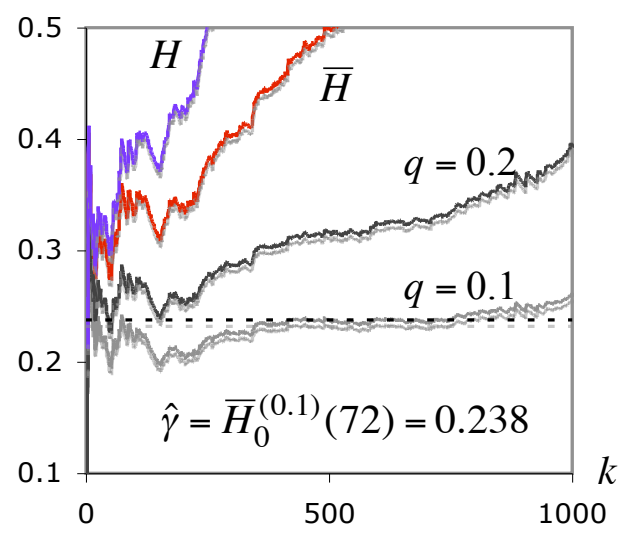

Figure 12: EVI-estimates, as function of $k$, for the MSFT data, as well as the adaptive estimates obtained through the Algorithm..

$\eta_{0}=402$ for the $\gamma$-estimate $0.24, k_{0}=72$ and the adaptive PORT-MVRB estimate $\hat{\gamma}=0.238$. The associated 99\% MVRB-confidence interval for $\gamma$ is $(0.183,0.342)$, with a size equal to 0.159 .

- The estimate of $k_{0}^{H}$, provided in $(13)$, is $\hat{k}_{0}^{H}=71$ and $H(71)=0.391$. The approximate $99 \%$ confidence interval is then $(0.286,0.518)$, with a size equal to 0.232 again larger than the size 0.159 obtained through the heuristic Algorithm in this paper.

\subsubsection{A simulated data set}

Due to the specificity of the log-returns, often modelled by a Student- $t$ or its skewed versions (see Jones and Faddy, 2003, and McNeill et al., 2005), we have arbitrarily simulated a random sample of size $n=1762$, from a Student's $t_{\nu}$-model with $\nu=4$ degrees of freedom. Figure 13 is equivalent to Figure 10, but for the Student $t_{4}$ generated sample.

- The number of positive elements is now $n_{0}=904$. We were led to the $\rho$-estimate $\hat{\rho} \equiv \hat{\rho}_{0}=-0.72$, obtained at the level $k_{1}=\left[n_{0}^{0.999}\right]=897$. The associated $\beta$-estimate was $\hat{\beta} \equiv \hat{\beta}_{0}=1.02$.

- In Step 5. of Algorithm I, we have been led to the choice $q_{0}=0.1$, associated with a run of size 667 of a $\gamma$-estimate equal to 0.2. In Step 6. of the Algorithm I, we 


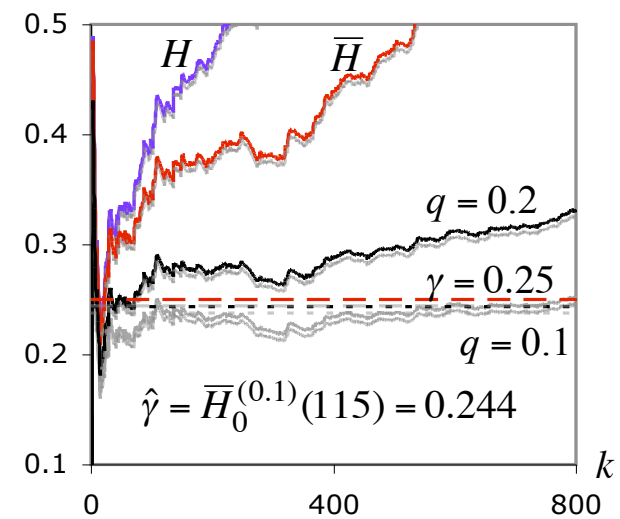

Figure 13: EVI-estimates, as function of $k$, associated with an underlying Student $t_{4}$ model, as well as the adaptive estimates obtained through the Algorithm.

got a mode $\eta_{0}=361$ for the $\gamma$-estimate $0.24, k_{0}=115$ and the adaptive PORTMVRB estimate $\hat{\gamma}=0.244$. The associated $99 \%$ MVRB-confidence interval for $\gamma$ is $(0.197,0.321)$, with a size equal to 0.124 .

- The estimate of $k_{0}^{H}$, provided in $(13)$, is $\hat{k}_{0}^{H}=73$ and $H(73)=0.359$. The approximate $99 \%$ confidence interval is then $(0.257,0.452)$, with a size equal to 0.195 .

- In this case we know the true value of $\gamma$, the value 0.25 , and we see that such a value does not even belong to the $99 \%$ confidence interval associated with the Hill estimate. On the other way round, it belongs to the $99 \%$ CI associated with the adaptive PORT-MVRB estimate. Moreover, the fact that the size of the MVRBCI is always smaller than the size of the Hill-CI, even when we consider both the classical and the PORT-MVRB estimators at the level $\hat{k}_{0}^{H}$, in (13), favours the new methodology.

\subsubsection{A general summary of the performed data analysis}

In Table 4, we present a summary of the data analysis performed in this section, providing the estimates of $k$ for the Hill EVI-estimation and the values of $\left(k_{0}, q_{0}\right)$ provided by the Algorithm considered. We also provide not only the most usual adaptive EVI-estimation of 
a positive $\gamma$, done through the classical Hill estimator, in (2), computed at the estimated level $\hat{k}_{0}^{H}$, in (13), as well as the adaptive EVI-estimation provided by the Algorithm in Section 4.1, related with the PORT-MVRB EVI estimators discussed in this article.

\begin{tabular}{|c|c|c|c|c|c|c|c|c|}
\hline Data & $n$ & $n_{0}$ & $k_{1}$ & $\hat{k}_{0}^{H}$ & $q_{0}$ & $k_{0}$ & $\hat{\gamma}:=\bar{H}_{0}^{\left(q_{0}\right)}\left(k_{0}\right)$ & $\hat{\gamma}^{H}:=H\left(\hat{k}_{0}^{H}\right)$ \\
\hline SECURA & 371 & 371 & 368 & 54 & $-1 / n$ & 233 & $0.255(0.218,0.307)$ & $0.320(0.219,0.420)$ \\
MSFT & 1762 & 882 & 876 & 71 & 0.1 & 72 & $0.238(0.183,0.342)$ & $0.391(0.286,0.518)$ \\
STU1 & 1762 & 904 & 897 & 73 & 0.1 & 115 & $0.244(0.197,0.321)$ & $0.359(0.257,0.452)$ \\
\hline
\end{tabular}

Table 4: Adaptive EVI-estimates for the different data sets under analysis.

For the SECURA data, and as desired, the adaptive PORT-MVRB EVI-estimate is inside the 99\% CI associated with the adaptive Hill EVI-estimate. But nothing similar happens with the other adaptive estimates. For the generated Student sample, the true value of $\gamma$ does not even belong to the $99 \%$ CI associated with the adaptive Hill EVIestimate. Moreover, $\hat{\gamma}$ is below the lower limit of the $99 \%$ CI associated with the adaptive Hill estimate, as well as the adaptive Hill estimate is above the upper limit of the $99 \%$ CI associated with the adaptive PORT-MVRB EVI-estimate. A similar comment applies to the MSFT data, and we strongly believe that in this case the true value of $\gamma$ is slightly below 0.286. These case studies claim obviously for a simulation study of the Algorithm presented in Section 4.1, but this is a topic out of the scope of this paper. However, the results obtained for other simulated samples, clearly indicate an over-estimation of the adaptive Hill estimate and an overall best performance of this data-driven (and locationinvariant) method of estimation of the extreme value index.

\section{References}

[1] Araújo Santos, P., Fraga Alves, M. I. and Gomes, M. I. (2006). Peaks over random threshold methodology for tail index and quantile estimation. Revstat 4:3, 227-247. 
[2] Beirlant, J., Goegebeur, Y., Segers, J. and Teugels, J. (2004). Statistics of Extremes. Theory and Applications. Wiley.

[3] Beirlant, J., Figueiredo, F., Gomes, M.I. and Vandewalle, B. (2008). Improved reduced-bias tail index and quantile estimators. J. Statist. Plann. and Inference 138:6, 1851-1870.

[4] Caeiro, F. and Gomes, M.I. (2006). A new class of estimators of a "scale" second order parameter Extremes 9, 193-211, 2007.

[5] Caeiro, F., Gomes, M.I. and Pestana, D.D. (2005). Direct reduction of bias of the classical Hill estimator. Revstat 3:2, 111-136.

[6] Caeiro, F., Gomes, M.I. and Henriques-Rodrigues, L. (2009). Reduced-bias tail index estimators under a third order framework. Commun. in Statist. - Theory and Methods 38:7, 1019-1040.

[7] Ciuperca, G. and Mercadier, C. (2010). Semi-parametric estimation for heavy tailed distributions. Extremes 13:1, 55-87.

[8] Drees, H. (2003). Extreme quantile estimation for dependent data, with applications to finance. Bernoulli 9:4, 617-657.

[9] Figueiredo, F., Gomes, M.I., Henriques-Rodrigues, L. and Miranda, M.C. (2010). A Computational Study of a Quasi-PORT Methodology for VaR Based on Second-Order Reduced-Bias Estimation. Notas e Comunicações CEAUL 05/2010. Submitted.

[10] Fraga Alves, M. I., Gomes M. I. and de Haan, L. (2003). A new class of semi-parametric estimators of the second order parameter. Portugaliae Mathematica 60:2: 194-213.

[11] Goegebeur, Y., Beirlant, J. and de Wet, T. (2008). Linking Pareto-tail kernel goodness-of-fit statistics with tail index at optimal threshold and second order estimation. Revstat 6:1, 5169 .

[12] Goegebeur, Y., Beirlant, J. and de Wet, T. (2010). Kernel estimators for the second order parameter in extreme value statistics. J. Statist. Plann. Inference 140:9, 2632-2654.

[13] Gomes, M.I. and Martins, M.J. (2002). "Asymptotically unbiased" estimators of the tail index based on external estimation of the second order parameter. Extremes 5:1, 5-31.

[14] Gomes, M.I. and Oliveira, O. (2001). The bootstrap methodology in Statistics of Extremes: choice of the optimal sample fraction. Extremes 4:4, 331-358, 2002. 
[15] Gomes, M. I. and Pestana, D. (2007). A simple second order reduced-bias' tail index estimator. J. Statist. Comp. and Simul. 77:6, 487-504.

[16] Gomes, M.I., Figueiredo, F. and Mendonça, S. (2004). Asymptotically best linear unbiased tail estimators under a second order regular variation. J. Statist. Plann. Inference 134:2, 409-433.

[17] Gomes, M.I., Fraga Alves, M.I. and Araújo Santos, P. (2008a). PORT Hill and Moment Estimators for Heavy-Tailed Models. Commun. Statist.: Simulation and Computation 37, 1281-1306.

[18] Gomes, M. I., de Haan, L. and Henriques-Rodrigues, L. (2008b). Tail Index estimation for heavy-tailed models: accommodation of bias in weighted log-excesses. J. Royal Statist. Soc. B70:1, 31-52.

[19] Gomes, M.I., Henriques-Rodrigues, L., Pereira, H. and Pestana, D. (2010). Tail index and second order parameters' semi-parametric estimation based on the log-excesses. J. Statist. Comput. and Simul. 80:6, 653-666.

[20] de Haan, L. and Peng, L. (1998). Comparison of tail index estimators. Statistica Neerlandica, 52, 60-70.

[21] Hall, P. (1982). On some simple estimates of an exponent of regular variation. J. Royal Statist. Soc. B44, 37-42.

[22] Hill, B. (1975). A simple general approach to inference about the tail of a distribution. Ann. Statist. 3, 1163-1174.

[23] Jones, M.C. and Faddy M.J. (2003). A skew extension of the $t$-distribution, with applications. J. Royal Statist. Soc. B 65:1, 159-174.

[24] McNeil, A., Frey R. and Embrechts, P. (2005). Quantitative Risk Management: Concepts, Techniques and Tools. Princeton University Press.

[25] Vandewalle, B. and Beirlant, J. (2006). On univariate extreme value statistics and the estimation of reinsurance premiums. Insurance Mathematics and Economics 38:3, 444-459. 